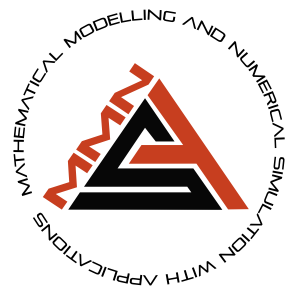

\title{
Fractional-order mathematical modelling of cancer cells-cancer stem cells-immune system interaction with chemotherapy
}

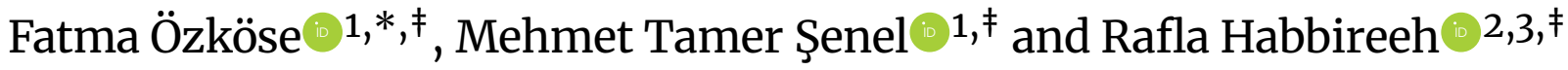 \\ ${ }^{1}$ Department of Mathematics, Faculty of Science, Erciyes University, Kayseri 38039, Turkey, ${ }^{2}$ Institute of Science, \\ Erciyes University, Kayseri 38039, Turkey, ${ }^{3}$ Department of Mathematics, Faculty of Science, Misurata University, \\ Misurata, Libya \\ *Corresponding Author \\ †fpeker@erciyes.edu.tr (Fatma Özköse); senel@erciyes.edu.tr (Mehmet Tamer Şenel); r.habbireeh@sci.misuratau.edu.ly (Rafla \\ Habbireeh)
}

\begin{abstract}
In this paper, we present a mathematical model of stem cells and chemotherapy for cancer treatment, in which the model is represented by fractional order differential equations. Local stability of equilibrium points is discussed. Then, the existence and uniqueness of the solution are studied. In addition, in order to point out the advantages of the fractional order modeling, the memory trace and hereditary traits are taken into consideration. Numerical simulations have been used to investigate how the fractional order derivative and different parameters affect the population dynamics, the graphs have been illustrated according to different values of fractional order $\alpha$ and different parameter values. Moreover, we have examined the effect of chemotherapy on tumor cells and stem cells over time. Furthermore, we concluded that the memory effect occurs as the $\alpha$ decreases from 1 and the chemotherapy drug is quite effective on the populations. We hope that this work will contribute to helping medical scientists take the necessary measures during the screening process and treatment.
\end{abstract}

Key words: Fractional-order differential equations; cancer stem cells; immune system; numerical solutions; memory effect; existence and uniqueness

AMS 2020 Classification: 92D25;26A33; 34A08

\section{Introduction}

Cancer is a general term that includes a wide range of diseases that can affect any part of the body. One of the distinguishing features of cancer is the rapid generation of abnormal cells that grow outside their normal limits and can then invade neighboring parts of the body and spread to other parts of it. Despite the scientific and technological development, cancer is a major cause of death worldwide, and it claimed the lives of 10 million people in 2020. According to the World Health Organization (WHO), between $30 \%$ and $50 \%$ of cancer cases can be prevented by avoiding risk factors for the disease to prevent it. The burden of cancer can also be reduced by detecting the disease early and providing patients with adequate treatment and care, given that the chances of recovery from many types of cancer increase if they are diagnosed early and treated appropriately. Many researchers have described the interactions between the immune system especially effector cells and tumor cells, where a mathematical modeling was used to clarify the relationship between them as in $[1,2,3,30,31]$. Recently, researches were directed to study the effect of stem cell therapy to reduce the growth of tumor cells due to the importance of stem cells in blood formation, as they grow into different types of blood cells such as red and white blood cells and platelets that contribute to stimulating

> Received: 13.11.2021 > Revised: 11.12.2021 > Accepted: 13.12.2021 > Published: 13.12.2021 
the patient's immune system and that were destroyed by Chemotherapy, radiotherapy, or both. A fractional-order model of tumor-immune system interaction has been proposed in [4], and a Chaotic dynamics of a fractional order HIV-1 model involving AIDS-related cancer cells has been given to understand the mechanism that underlies AIDS-related cancers in [8]. As in $[13,14,15,16]$ the effectiveness of using stem cells to boost the patient's immune system has been shown, which may in the future be a treatment for most types of cancer. In this work, we extend the study [17]. Taking into account the interaction of stem cells, tumor cells and chemotherapy for the treatment of cancer, we propose a fractional-order instead of integer-order model to show how effective stem cells are in improving the immune system, which in turn better fights tumor cells [10,11, 12, 18]. Many real life systems are described better by fractional differential equations, e.g. heat equation, telegraph equation, social systems, medical imaging, pollution control, cancer dynamics, infectious diseases, and a lossy electric transmission line are all involved with fractional order operators $[8,9,18,19]$. We propose a model motivated by Manar A. Alqudah's work [17], Manar presented a study of ordinary differential equations model that describes the stem cells and chemotherapy for treatment of cancer to show how the stem cells support the effector cells which fighting the tumor cells to improve the immune system of the cancer patient while the chemotherapy kills the infected cells. The mathematical model of treatment of cancer studied in [17] is presented by:

$$
\left\{\begin{array}{l}
\frac{d S}{d t}=\gamma_{1} S-k_{s} M S, \\
\frac{d T}{d t}=r(1-b T) T-\left(p_{3} E+k_{T} M\right) T, \\
\frac{d E}{d t}=\sigma-\mu E+\frac{p_{1} E S}{S+1}-p_{2}(T+M) E, \\
\frac{d M}{d t}=-\gamma_{2} M+V(t) .
\end{array}\right.
$$

In the previous model $S(t)$ stem cells, $T(t)$ tumor cells, $E(t)$ effector cells, $M(t)$ chemotherapy concentration drug, and the initial conditions are: $S(0)=S_{0}, E(0)=E_{0}, T(0)=T_{0}, 0 \leq t \leq \infty$ and $M(0)=0$ if $V_{0}=0$.

In our paper, the fractional order form of the model (1) is considered with the Caputo sense [20]. In addition, so that the system (1) is dimensionally consistent: the units of measurement from the left- and right-hand sides of the equations agree. It has been achieved by modifying the parameters involved in the right-hand side of the equations, e.g. raising them to power $\alpha$. The new system as follows:

$$
\left\{\begin{array}{l}
{ }_{C} D^{\alpha} S(t)=\gamma_{1}^{\alpha} S-k_{S}^{\alpha} M S \\
{ }_{C} D^{\alpha} T(t)=r^{\alpha}\left(1-b^{\alpha} T\right) T-\left(p_{3}^{\alpha} E+k_{T}^{\alpha} M\right) T \\
{ }_{C} D^{\alpha} E(t)=\sigma^{\alpha}-\mu^{\alpha} E+\frac{p_{1}^{\alpha} E S}{S+1}-p_{2}^{\alpha}(T+M) E \\
{ }_{C} D^{\alpha} M(t)=-\gamma_{2}^{\alpha} M+V(t),
\end{array}\right.
$$

with the same initial conditions in (1) and $\alpha$ is the order of the model $0<\alpha \leq 1$.

The parameters description are summarized in Table 1 . Some values are taken arbitrarily to easy solving the model numerically and the others are taken from [17] to be compatible with the description of model (2). We assumed that all of the parameters to be non-negative was $\gamma_{1}$ non-positive as stated in [17].

Table 1. Parameter values used for numerical analysis

\begin{tabular}{llll}
\hline Parameters & Description & Values & Reference \\
\hline$S_{0}$ & Stem cells initial concentration & 1 & {$[17]$} \\
$T_{0}$ & Density of free tumors & 1 & {$[17]$} \\
$E_{0}$ & Effector cells initial concentration & 1 & {$[17]$} \\
$M_{0}$ & Chemotherapy concentration drug & 1 & {$[17]$} \\
$V_{0}$ & The time dependent external influx of chemotherapy drug & 0.18 & {$[17]$} \\
$r$ & Tumor growth rate & 0 & {$[17]$} \\
$\gamma_{1}$ & Decay rate of concentration of stem cells & -0.02825 & {$[17]$} \\
$\gamma_{2}$ & Decay rate of chemotherapy drug & 6.4 & {$[17]$} \\
$\sigma$ & The rate of produced effector cells & 0.17 & {$[17]$} \\
$\mu$ & The natural death rate of the effector cell & 0.03 & {$[17]$} \\
$p_{1}$ & Maximum rate of effector cells & 0.1245 & {$[17]$} \\
$p_{2}$ & Decay rate of effector cells killed by tumor cells and chemotherapy & 1 & {$[17]$} \\
$p_{3}$ & Decay rate of tumor cells killed by effector cells & 0.9 & {$[17]$} \\
$b$ & Carrying capacity of tumor cells & $10^{-9}$ & {$[17]$} \\
$k_{s}$ & Fractional stem cells killed by chemotherapy & 1 & {$[17]$} \\
$k_{T}$ & Fractional tumor cells killed by chemotherapy & 0.9 & {$[17]$} \\
$V(t)$ & The time dependent external influx of chemotherapy drug & 1 & {$[17]$} \\
\hline
\end{tabular}

Motivated by the above discussion, the aim of this study is to investigate a fractional-order mathematical model of stem cell- cancer cell- immune system interaction. The reason of using fractional order differential equations is that they are naturally related to systems with memory which exists in cancer cells-immune system interactions. The most essential property of these models is their nonlocal property which does not exist in the integer order differential operators. Mathematical models, using ordinary differential equations with integer order have been proved valuable in understanding the dynamics of diseases. But, they have some limitations when compared with the fractional order derivatives. Integer order derivatives only describe the instantaneous biological events. Fractional order's nonlocal 
property says that the next stage of a model depends not only upon its current state but also upon all of its historical states. Therefore, models with fractional order differential equations provide more advantages than integer order mathematical models. In this study, both fractional modeling has been taken into account and estimated data have been used. Meanwhile, dimensional compatibility has been considered in order to better reveal the effect of fractional-order in the proposed fractional-order stem cell-cancer cell-immune system interaction. Additionally, we have aimed to point out the advantages of the fractional order modeling, taking into consideration the memory trace and hereditary traits which are capable of integrating all past activities and taking into account the long-term history of the system. In this context, it can be seen that the memory trace dynamics are highly dependent on time. When the fractional-order $\alpha$ is decreased from the unit, the memory trace nonlinearly increases from 0 . Hence, the fractional-order system dynamics are quite different from the integer-order dynamics. It is thought that there is no such study in the literature that deals with the stem cell-cancer relationship, and making the fractional order model dimensionally consistent, and taking into account the memory effect/hereditary characteristics.

The remaining part of this paper is prepared as follows. In Sec. (2), some definitions of a fractional order derivative (FOD) and some important theorems for FODs are given. In Sec. (3), the existence and uniqueness conditions of the solutions are given. In Sec. (4), stability theorems for the equilibrium points are examined. In Sec. (5), the numerical simulation and data analysis have been given. In Sec. (6), the effects of the memory trace on the behaviour of the system (2) are examined. In Sec. (7), to investigate the effects of different parameter values and different values of $\alpha$ on the dynamic behavior of the proposed model, the numerical solutions have been carried out. Finally, the Results and Discussion are given in Sec. (8).

\section{Preliminaries}

The fractional-order derivation and the fractional-order integration have many definitions such that the Riemann-Liouville definition, Caputo definition, Hadamard fractional integral, Atangana-Baleanu fractional integral, Riesz derivative, and Generalized Functions approaches $[5,7,20,26]$. The most commonly used of these are Riemann-Liouville and Caputo definitions. Caputo reformulated the definition of the Riemann-Liouville fractional derivative by switching the order of the ordinary derivative with the fractional integral operator. By doing so, the Laplace transform of this new derivative depends on integer order initial conditions, differently from the initial conditions when we use the Riemann-Liouville fractional derivative, which involves fractional order conditions, give a well understanding of the properties of many physical phenomena which makes it applicable to the problems of our real world.

Definition $1[20,26]$ The fractional integral of order $\alpha>0$, of the function $f(t), t>0$ is given by

$$
I^{\alpha} f(t)=\int_{0}^{t} \frac{(t-s)^{\alpha-1}}{\Gamma(\alpha)} f(s) d s
$$

and the fractional derivative of order $\alpha \in(n-1, n)$ of $f(t), t>0$ is given by

$$
D^{\alpha} f(t)=I^{n-\alpha} D^{n} f(t) \quad\left(D=\frac{d}{d t}\right) .
$$

Definition 2 [20] Let $f: R_{+} \rightarrow R$ continuous function. The Caputo fractional-order derivative is given by

$$
{ }_{C} D_{t_{0}, t}^{\alpha} f(t)=\frac{1}{\Gamma(m-\alpha)} \int_{0}^{t}(t-\tau)^{m-\alpha-1} f^{(m)}(\tau) d \tau .
$$

where $m-1<\alpha<m \in Z^{+}$. For the special case of $0<\alpha<1$, we have

$$
{ }_{C} D_{0, t}^{\alpha} f(t)=\frac{1}{\Gamma(1-\alpha)} \int_{0}^{t}(t-\tau)^{-\alpha} f^{\prime}(\tau) d \tau .
$$

For convenience, we use the notation ${ }_{C} D^{\alpha} f(t)$ instead of ${ }_{C} D_{0, t}^{\alpha} f(t)$ to denote the Caputo fractional-order derivative operator.

Theorem $1[27,28]$ If $X^{*}$ is the equilibrium point of system (2), then system (2) is

(1) Asymptotically stable $\Longleftrightarrow$ all the eigenvalues $\lambda_{i}, i=1,2, \ldots, n$ of the Jacobian matrix $J\left(X^{*}\right)$ satisfy that $\left|\arg \left(\lambda_{i}\right)\right|>\frac{\alpha \pi}{2}$.

(2) Stable $\Longleftrightarrow$ it is asymptotically stable or the eigenvalues $\lambda_{i}, i=1,2, \ldots$, nof $J\left(X^{*}\right)$ that satisfy $\left|\arg \left(\lambda_{i}\right)\right|=\frac{\alpha \pi}{2}$ have the same geometric and geometric multiplicity for $\lambda_{i}$ is 1 .

(3) Unstable $\Longleftrightarrow$ eigenvalues $\lambda_{i}$ for some $i=1,2, \ldots$, nof $J\left(X^{*}\right) \operatorname{satisfy}\left|\arg \left(\lambda_{i}\right)\right|<\frac{\alpha \pi}{2}$.

\section{Existence and uniqueness}

Consider system (2) with the initial conditions $S(0)=S_{0}, E(0)=E_{0}, T(0)=T_{0}, M(0)=0$ if $V_{0}=0$. System (2) can be written in the following form:

$$
\left\{\begin{aligned}
{ }_{C} D^{\alpha} X(t) & =B_{1} X(t)+S(t) B_{2} X(t)+T(t) B_{3} X(t)+E(t) B_{4} X(t)+M(t) B_{5} X(t)+\vartheta \\
X\left(t_{0}\right) & =X_{0}
\end{aligned}\right.
$$


where

$$
\begin{gathered}
X(t)=\left(\begin{array}{l}
S(t) \\
T(t) \\
E(t) \\
M(t)
\end{array}\right), X(0)=\left(\begin{array}{c}
S(0) \\
T(0) \\
E(0) \\
M(0)
\end{array}\right), B_{1}=\left(\begin{array}{cccc}
\gamma_{1}^{\alpha} & 0 & 0 & 0 \\
0 & r^{\alpha} & 0 & 0 \\
0 & 0 & -\mu^{\alpha} & 0 \\
0 & 0 & 0 & -\gamma_{2}^{\alpha}
\end{array}\right), \\
B_{2}=\left(\begin{array}{llll}
0 & 0 & 0 & -k_{S}^{\alpha} \\
0 & 0 & 0 & 0 \\
0 & 0 & 0 & 0 \\
0 & 0 & 0 & 0
\end{array}\right), B_{3}=\left(\begin{array}{cccc}
0 & 0 & 0 & 0 \\
0 & -b^{\alpha} & 0 & 0 \\
0 & 0 & -p_{2}^{\alpha} & 0 \\
0 & 0 & 0 & 0
\end{array}\right), B_{4}=\left(\begin{array}{cccc}
0 & 0 & 0 & 0 \\
0 & -p_{3}^{\alpha} & 0 & 0 \\
p_{1}^{\alpha} & 0 & 0 & 0 \\
S+1 & 0 & 0 & 0 \\
0 & 0 & & \\
&
\end{array}\right), \\
B_{5}=\left(\begin{array}{cccc}
0 & 0 & 0 & 0 \\
0 & -k_{T}^{\alpha} & 0 & 0 \\
0 & 0 & -p_{2}^{\alpha} & 0 \\
0 & 0 & 0 & 0
\end{array}\right), \vartheta=\left(\begin{array}{c}
0 \\
0 \\
\sigma^{\alpha} \\
V(t)
\end{array}\right) .
\end{gathered}
$$

In view of $[4,25,26]$ desired definitions for the existence and uniqueness are defined as follows:

Definition 3 Let $C^{*}[0, \tau]$ be the class of continuous column vector $X(t)$ whose components $S, T, E, M \in C^{*}[0, \tau]$ are the class of continuous functions on the interval $[0, \tau]$. The norm of $X \in C^{*}[0, \tau]$ is given by

$$
\|X\|=\sup _{t}\left|e^{-N t} S(t)\right|+\sup _{t}\left|e^{-N t} T(t)\right|+\sup _{t}\left|e^{-N t} E(t)\right|+\sup _{t}\left|e^{-N t} M(t)\right|,
$$

where $N$ is a natural number and when $t>\delta \geq m$, we write $C_{\delta}^{*}[0, \tau]$ and $C_{\delta}[0, \tau]$.

Definition $4 X \in C^{*}[0, \tau]$ is a solution of IVP (3) if

(1) $(t, X(t)) \in \mathcal{D}, t \in[0, \tau]$ where $\mathcal{D}=[0, \tau] \times \mathcal{K}, \mathcal{K}=\left\{(S, T, E, M) \in \mathcal{R}_{+}^{4}:|S| \leq p, \quad|T| \leq r,|E| \leq w,|M| \leq q\right\} ; p, r, w, q \in R_{+}$are constants.

(2) $X(t)$ satisfies (3).

Theorem 2 The solution $X$ of IVP (3) is unique and $X \in C^{*}[0, \tau]$.

Proof From the properties of fractional calculus, Eq. (3) can be written as

$$
I^{1-\alpha} \frac{d}{d t} X(t)=B_{1} X(t)+S(t) B_{2} X(t)+T(t) B_{3} X(t)+E(t) B_{4} X(t)+M(t) B_{5} X(t)+\vartheta .
$$

Operating by $I^{\alpha}$, we obtain

$$
X(t)=X(0)+I^{\alpha}\left(B_{1} X(t)+S(t) B_{2} X(t)+T(t) B_{3} X(t)+E(t) B_{4} X(t)+M(t) B_{5} X(t)+\vartheta\right)
$$

Now let $F: C^{*}[0, \tau] \rightarrow C^{*}[0, \tau]$ defined by

$$
F X(t)=X(0)+I^{\alpha}\left(B_{1} X(t)+S(t) B_{2} X(t)+T(t) B_{3} X(t)+E(t) B_{4} X(t)+M(t) B_{5} X(t)+\vartheta\right) .
$$

Then

$$
\begin{aligned}
e^{-N t}(F X-F Y) & =e^{-N t} I^{\alpha}\left(B_{1}(X(t)-Y(t))+S(t) B_{2}(X(t)-Y(t))+T(t) B_{3}(X(t)-Y(t))+E(t) B_{4}(X(t)-Y(t))+M(t) B_{5}(X(t)-Y(t))\right) \\
& \leq\left|\frac{1}{\Gamma(\alpha)} \int_{0}^{t}(t-s)^{\alpha-1} e^{-N(t-s)}(X(s)-Y(s)) e^{-N s} d s\right|\left(B_{1}+p B_{2}+r B_{3}+w B_{4}+q B_{5}\right) \\
& \leq \frac{\left(B_{1}+p B_{2}+r B_{3}+w B_{4}+q B_{5}\right)|\gamma(\alpha, u)|}{N^{\alpha}}\|X-Y\|,
\end{aligned}
$$

where $\gamma(\alpha, u)$ is the lower incomplete gamma function and $u=t-s$. If we choose $N$ such that $N^{\alpha} \geq|\gamma(\alpha, u)| B_{1}+p B_{2}+r B_{3}+w B_{4}+q B_{5}$, then we obtain $\|F X-F Y\| \leq\|X-Y\|$. Operator $F$ in (5) has a fixed point. Thus, (4) has a unique solution $X \in C^{*}[0, \tau]$. From (4) we have

$$
\begin{aligned}
X(t) & =X(0)+\frac{t^{\alpha}}{\Gamma(\alpha+1)}\left(B_{1} X(0)+S(0) B_{2} X(0)+T(0) B_{3} X(0)+E(0) B_{4} X(0)+M(0) B_{5} X(0)+\vartheta\right)+I^{\alpha+1}\left(B_{1} X^{\prime}(t)+S^{\prime}(t) B_{2} X(t)\right. \\
& +S(t) B_{2} X^{\prime}(t)+T^{\prime}(t) B_{3} X(t)+T(t) B_{3} X^{\prime}(t)+E^{\prime}(t) B_{4} X(t)+E(t) B_{4} X^{\prime}(t)+M^{\prime}(t) B_{5} X(t)+M(t) B_{5} X^{\prime}(t) . \\
e^{-N t} X^{\prime} & =e^{-N t}\left[\frac{t^{\alpha-1}}{\Gamma(\alpha)}\left(B_{1} X(0)+S(0) B_{2} X(0)+T(0) B_{3} X(0)+E(0) B_{4} X(0)+M(0) B_{5} X(0)+\vartheta\right)+I^{\alpha}\left(B_{1} X^{\prime}(t)+S^{\prime}(t) B_{2} X(t)\right.\right. \\
& \left.+S(t) B_{2} X^{\prime}(t)+T^{\prime}(t) B_{3} X(t)+T(t) B_{3} X^{\prime}(t)+E^{\prime}(t) B_{4} X(t)+E(t) B_{4} X^{\prime}(t)+M^{\prime}(t) B_{5} X(t)+M(t) B_{5} X^{\prime}(t)\right] .
\end{aligned}
$$


from which we can deduce that $X^{\prime} \in C_{\sigma}^{*}[0, \tau]$. From (4) we get

$$
\frac{d X}{d t}=\frac{d}{d t} I^{\alpha}\left(B_{1} X(t)+S(t) B_{2} X(t)+T(t) B_{3} X(t)+E(t) B_{4} X(t)+M(t) B_{5} X(t)+\vartheta\right) .
$$

Operating by $I^{1-\alpha}$ we get

$$
\begin{aligned}
I^{1-\alpha} \frac{d X}{d t} & =I^{1-\alpha} \frac{d}{d t} I^{\alpha}\left(B_{1} X(t)+S(t) B_{2} X(t)+T(t) B_{3} X(t)+E(t) B_{4} X(t)+M(t) B_{5} X(t)+V(t) C+\vartheta\right) . \\
{ }_{C} D^{\alpha} X(t) & \left.=B_{1} X(t)+S(t) B_{2} X(t)+T(t) B_{3} X(t)+E(t) B_{4} X(t)+M(t) B_{5} X(t)+\vartheta\right),
\end{aligned}
$$

and

$$
X(0)=X_{0}+I^{\alpha}\left(B_{1} X(t)+S(t) B_{2} X(t)+T(t) B_{3} X(t)+E(t) B_{4} X(t)+M(t) B_{5} X(t)+\vartheta\right)
$$

Therefore, Eq. (4) is equivalent to IVP (3).

\section{Equilibrium points and stability analysis}

To calculate the equilibrium points of system (2) let [29]

$$
\left\{\begin{array}{l}
{ }_{C} D^{\alpha} S(t)=0 \\
{ }_{C} D^{\alpha} T(t)=0 \\
{ }_{C} D^{\alpha} E(t)=0 \\
{ }_{C} D^{\alpha} M(t)=0
\end{array}\right.
$$

Thus,

$$
\left\{\begin{array}{l}
\gamma_{1}^{\alpha} S-k_{s}^{\alpha} M S=0 \\
r^{\alpha}\left(1-b^{\alpha} T\right) T-\left(p_{3}^{\alpha} E+k_{T}^{\alpha} M\right) T=0 \\
\sigma^{\alpha}-\mu^{\alpha} E+\frac{p_{1}^{\alpha} E S}{S+1}-p_{2}^{\alpha}(T+M) E=0 \\
-\gamma_{2}^{\alpha} M+V(t)=0
\end{array}\right.
$$

Then the equilibrium points are:

$$
\begin{aligned}
E q_{1}=\left(S_{1}, T_{1}, E_{1}, M_{1}\right)= & \left(0,0, \frac{\sigma^{\alpha} \gamma_{2}^{\alpha}}{p_{2}^{\alpha} V+\gamma_{2}^{\alpha} \mu^{\alpha}}, \frac{V}{\gamma_{2}^{\alpha}}\right), \\
E q_{2}=\left(S_{2}, T_{2}, E_{2}, M_{2}\right)= & \left(0, \frac{p_{2}^{\alpha} V\left(r^{\alpha} b^{\alpha}-k_{T}^{\alpha}\right)+r^{\alpha} \gamma_{2}^{\alpha}\left(p_{2}^{\alpha}-b^{\alpha} \mu^{\alpha}\right)-\sqrt{-4 \sigma^{\alpha} b^{\alpha} p_{2}^{\alpha} p_{3}^{\alpha} r^{\alpha} \gamma_{2}^{2 \alpha}+a^{2}}}{2 p_{2}^{\alpha} \gamma_{2}^{\alpha} r^{\alpha} b^{\alpha}},\right. \\
& \left.\frac{p_{2}^{\alpha} V\left(r^{\alpha} b^{\alpha}-k_{T}^{\alpha}\right)+r^{\alpha} \gamma_{2}^{\alpha}\left(p_{2}^{\alpha}+b^{\alpha} \mu^{\alpha}\right)+\sqrt{-4 \sigma^{\alpha} b^{\alpha} p_{2}^{\alpha} p_{3}^{\alpha} r^{\alpha} \gamma_{2}^{2 \alpha}+a^{2}}}{2 p_{2}^{\alpha} p_{3}^{\alpha} \gamma_{2}^{\alpha}}, \frac{V}{\gamma_{2}^{\alpha}}\right), \\
E q_{3}=\left(S_{3}, T_{3}, E_{3}, M_{3}\right)= & \left(0, \frac{p_{2}^{\alpha} V\left(r^{\alpha} b^{\alpha}-k_{T}^{\alpha}\right)+r^{\alpha} \gamma_{2}^{\alpha}\left(p_{2}^{\alpha}-b^{\alpha} \mu^{\alpha}\right)+\sqrt{-4 \sigma^{\alpha} b^{\alpha} p_{2}^{\alpha} p_{3}^{\alpha} r^{\alpha} \gamma_{2}^{2 \alpha}+a^{2}}}{2 p_{2}^{\alpha} \gamma_{2}^{\alpha} r^{\alpha} b^{\alpha}},\right. \\
& \left.\frac{p_{2}^{\alpha} V\left(r^{\alpha} b^{\alpha}-k_{T}^{\alpha}\right)+r^{\alpha} \gamma_{2}^{\alpha}\left(p_{2}^{\alpha}+b^{\alpha} \mu^{\alpha}\right)-\sqrt{-4 \sigma^{\alpha} b^{\alpha} p_{2}^{\alpha} p_{3}^{\alpha} r^{\alpha} \gamma_{2}^{2 \alpha}+a^{2}}}{2 p_{2}^{\alpha} p_{3}^{\alpha} \gamma_{2}^{\alpha}}, \frac{V}{\gamma_{2}^{\alpha}}\right),
\end{aligned}
$$

where $a=\left(\left(k_{T}^{\alpha}-r^{\alpha} b^{\alpha}\right) p_{2}^{\alpha} V-\gamma_{2}^{\alpha} r^{\alpha}\left(p_{2}^{\alpha}+b^{\alpha} \mu^{\alpha}\right)\right.$, and equilibrium points must verify $E q_{1}, E q_{2}, E q_{3}>0$. Hence, $p_{2}^{\alpha}, p_{3}^{\alpha}, \mu^{\alpha}, \gamma_{2}^{\alpha}, \sigma^{\alpha}, r^{\alpha}, b^{\alpha}, V, k_{T}^{\alpha} \in$ $D_{1}$, where $\mathcal{D}_{1}=\left\{\left(p_{2}^{\alpha}, p_{3}^{\alpha}, \mu^{\alpha}, \gamma_{2}^{\alpha}, \sigma^{\alpha}, r^{\alpha}, b^{\alpha}, V, k_{T}^{\alpha}\right) \in \mathcal{R}_{+}^{9}: V k_{T}^{\alpha}-r^{\alpha}\left(b^{\alpha}+\gamma_{2}^{\alpha}\right)+\sigma^{\alpha} \gamma_{2}^{\alpha}>0\right\} \cap\left\{\left(p_{2}^{\alpha}, p_{3}^{\alpha}, \mu^{\alpha}, \gamma_{2}^{\alpha}, \sigma^{\alpha}, r^{\alpha}, b^{\alpha}, V, k_{T}^{\alpha}\right) \in \mathcal{R}_{+}^{9}:\right.$ $\left.\left(V p_{2}^{\alpha}\left(k_{T}^{\alpha}-r^{\alpha} b^{\alpha}\right)-r^{\alpha} \gamma_{2}^{\alpha}\left(p_{2}^{\alpha}+b^{\alpha} \mu^{\alpha}\right)\right)^{2} \geq 4 \sigma^{\alpha} b^{\alpha} p_{2}^{\alpha} p_{3}^{\alpha} \gamma_{2}^{2 \alpha}\right\}$.

Theorem 3 Let $E q_{1} \in D_{1}$ be the equilibrium point of system (2) and the following conditions are valid: $\gamma_{1}^{\alpha} \gamma_{2}^{\alpha}<k_{s}^{\alpha} V,\left(p_{2}^{\alpha} V+\gamma_{2}^{\alpha} \mu^{\alpha}\right)\left(\gamma_{2}^{\alpha} r^{\alpha}+k_{T}^{\alpha} V\right)<p_{3}^{\alpha} \sigma^{\alpha} \gamma_{2}^{2 \alpha}$ and $p_{2}^{\alpha} V+\gamma_{2}^{\alpha} \mu^{\alpha}>0$.

Then $E q_{1}$ is locally asymptotically stable.

Proof The Jacobian matrix of the model (2) at $E q_{1}$ is

$$
J\left(E q_{1}\right)=\left(\begin{array}{cccc}
\gamma_{1}^{\alpha}-k_{s}^{\alpha} \frac{V}{\gamma_{2}^{\alpha}} & 0 & 0 & 0 \\
0 & r^{\alpha}-p_{3}^{\alpha} \frac{\sigma^{\alpha} \gamma_{2}^{\alpha}}{p_{2}^{\alpha} V+\gamma_{2}^{\alpha} \mu^{\alpha}}+k_{T}^{\alpha} \frac{V}{\gamma_{2}^{\alpha}} & 0 & 0 \\
p_{1}^{\alpha} \frac{\sigma^{\alpha} \gamma_{2}^{\alpha}}{p_{2}^{\alpha} V+\gamma_{2}^{\alpha} \mu^{\alpha}} & -p_{2}^{\alpha} \frac{\sigma^{\alpha} \gamma_{2}^{\alpha}}{p_{2}^{\alpha} V+\gamma_{2}^{\alpha} \mu^{\alpha}} & -\mu^{\alpha}-p_{2}^{\alpha} \frac{V}{\gamma_{2}^{\alpha}} & -p_{2}^{\alpha} \frac{\sigma^{\alpha} \gamma_{2}^{\alpha}}{p_{2}^{\alpha} V+\gamma_{2}^{\alpha} \mu^{\alpha}} \\
0 & 0 & 0 & -\gamma_{2}^{\alpha}
\end{array}\right) .
$$

The characteristic equation is $\left|J\left(E q_{1}\right)-\lambda I\right|=0$. Hence, $\left(\gamma_{1}^{\alpha}-k_{s}^{\alpha} \frac{V}{\gamma_{2}^{\alpha}}-\lambda\right)\left(r^{\alpha}-p_{3}^{\alpha} \frac{\sigma^{\alpha} \gamma_{2}^{\alpha}}{p_{2}^{\alpha} V+\gamma_{2}^{\alpha} \mu^{\alpha}}+k_{T}^{\alpha} \frac{V}{\gamma_{2}^{\alpha}}-\lambda\right)\left(-\gamma_{2}^{\alpha}-\lambda\right)\left(-\mu^{\alpha}-p_{2}^{\alpha} \frac{V}{\gamma_{2}^{\alpha}}-\lambda\right)=0$. 
Eigenvalues of $J\left(E q_{1}\right)$ are $\lambda_{1}=\gamma_{1}^{\alpha}-k_{s}^{\alpha} \frac{V}{\gamma_{2}^{\alpha}}, \lambda_{2}=r^{\alpha}-p_{3}^{\alpha} \frac{\sigma^{\alpha} \gamma_{2}^{\alpha}}{p_{2}^{\alpha} V+\gamma_{2}^{\alpha} \mu^{\alpha}}-k_{T}^{\alpha} \frac{V}{\gamma_{2}^{\alpha}}, \lambda_{3}=-\mu^{\alpha}-p_{2}^{\alpha} \frac{V}{\gamma_{2}^{\alpha}}, \lambda_{4}=-\gamma_{2}^{\alpha}$. From the conditions we have $\lambda_{i}<0$ for $i=1,2,3,4$. Therefore, $\left|\arg \left(\lambda_{i}\right)\right|>\frac{\alpha \pi}{2}$. By Theorem (1), $E q_{1}$ is locally asymptotically stable.

Theorem 4 Let $E q_{2} \in D_{1}$ be the equilibrium point of system (2) and $\left(p_{2}^{\alpha}, p_{3}^{\alpha}, \mu^{\alpha}, \gamma_{2}^{\alpha}, \sigma^{\alpha}, r^{\alpha}, b^{\alpha}, V, k_{T}^{\alpha}\right) \in D_{1} \cap\left(Q^{*} \cup P^{*}\right)$, where $Q^{*}=\left\{\left(p_{2}^{\alpha}, p_{3}^{\alpha}, \mu^{\alpha}, \gamma_{2}^{\alpha}, \sigma^{\alpha}, r^{\alpha}, b^{\alpha}, V, k_{T}^{\alpha}\right) \in R_{+}^{9}: d^{2}, d_{1} \geq 0, \gamma_{1}^{\alpha} \gamma_{2}^{\alpha}<k_{s}^{\alpha} V\right.$ and $\left(\left(2 b^{\alpha}+p_{2}^{\alpha}\right)\left(d+k_{T}^{\alpha} p_{2}^{\alpha} V\right) r^{\alpha} \gamma_{2}^{\alpha}-b^{\alpha} r^{3 \alpha} \gamma_{2}^{\alpha}\left(b^{\alpha} p_{2}^{\alpha} V-p_{2}^{\alpha} \gamma_{2}^{\alpha}+\right.\right.$ $\left.\left.\left.b^{\alpha} \gamma_{2}^{\alpha} \mu^{\alpha}\right)+r^{2 \alpha} \gamma_{2}^{\alpha}\left(-p_{2}^{2 \alpha} \gamma_{2}^{\alpha}+2 b^{2 \alpha}\left(p_{2}^{\alpha} V+\gamma_{2}^{\alpha} \mu^{\alpha}\right)-b^{\alpha}\left(d+p_{2}^{\alpha}\left(\left(k_{T}^{\alpha}+p_{2}^{\alpha}\right) V+\gamma_{2}^{\alpha}\left(2+\mu^{\alpha}\right)\right)\right)\right)-d_{1}\right)<0\right\} . P^{*}=\left\{\left(p_{2}^{\alpha}, p_{3}^{\alpha}, \mu^{\alpha}, \gamma_{2}^{\alpha}, \sigma^{\alpha}, r^{\alpha}, b^{\alpha}, V, k_{T}^{\alpha}\right) \in\right.$ $R_{+}^{9}: d^{2}$ or $d_{1}<0, \gamma_{1}^{\alpha} \gamma_{2}^{\alpha}<k_{S}^{\alpha} V$ and

$\operatorname{Re}\left(\left(\left(2 b^{\alpha}+p_{2}^{\alpha}\right)\left(d+k_{T}^{\alpha} p_{2}^{\alpha} V\right) r^{\alpha} \gamma_{2}^{\alpha}-b^{\alpha} r^{3 \alpha} \gamma_{2}^{\alpha}\left(b^{\alpha} p_{2}^{\alpha} V-p_{2}^{\alpha} \gamma_{2}^{\alpha}+b^{\alpha} \gamma_{2}^{\alpha} \mu^{\alpha}\right)+r^{2 \alpha} \gamma_{2}\left(-p_{2}^{2 \alpha} \gamma_{2}^{\alpha}+2 b^{2 \alpha}\left(p_{2}^{\alpha} V+\gamma_{2}^{\alpha} \mu^{\alpha}\right)-b^{\alpha}\left(d+p_{2}^{\alpha}\left(\left(k_{T}^{\alpha}+p_{2}^{\alpha}\right) V+\right.\right.\right.\right.\right.$ $\left.\left.\left.\left.\left.\left.\gamma_{2}^{\alpha}\left(2+\mu^{\alpha}\right)\right)\right)\right)-d_{1}\right)\right)<0\right\}$,

whered $=\sqrt{-4 \sigma^{\alpha} b^{\alpha} p_{2}^{\alpha} p_{3}^{\alpha} r^{\alpha} \gamma_{2}^{2 \alpha}+\left(k_{T}^{\alpha} p_{2}^{\alpha} V-b^{\alpha} p_{2}^{\alpha} r^{\alpha} V-p_{2}^{\alpha} r^{\alpha} \gamma_{2}^{\alpha}-b^{\alpha} r^{\alpha} \gamma_{2}^{\alpha} \mu^{\alpha}\right)^{2}}$ and $d_{1}=\left(r^{2 \alpha} \gamma_{2}^{2 \alpha}\left(\left(-p_{2}^{\alpha}\left(d+k_{T}^{\alpha} p_{2}^{\alpha} V-p_{2}^{\alpha} r^{\alpha} \gamma_{2}^{\alpha}\right)+b^{2 \alpha}(-2+\right.\right.\right.$ $\left.\left.r^{\alpha}\right) r^{\alpha}\left(p_{2}^{\alpha} V+\gamma_{2}^{\alpha} \mu^{\alpha}\right)+b^{\alpha}\left(d\left(-2+r^{\alpha}\right)-2 k_{T}^{\alpha} p_{2}^{\alpha} V+p_{2}^{\alpha} r^{\alpha}\left(\left(k_{T}^{\alpha}+p_{2}^{\alpha}\right) V+\gamma_{2}^{\alpha}\left(2-r^{\alpha}+\mu^{\alpha}\right)\right)\right)\right)^{2}-8 b^{\alpha} p_{2}^{\alpha}$

$\left(-k_{T}^{2 \alpha} p_{2}^{2 \alpha}\left(-2+r^{\alpha}\right) V^{2}-2 k_{T}^{\alpha} p_{2}^{\alpha} r^{\alpha} V\left(-p_{2}^{\alpha}\left(-2+r^{\alpha}\right) \gamma_{2}^{\alpha}+b^{\alpha}\left(p_{2}^{\alpha} V+\gamma_{2}^{\alpha} \mu^{\alpha}\right)\right)+r^{\alpha}\left(-p_{2}^{2 \alpha}\left(-2+r^{\alpha}\right) r^{\alpha} \gamma_{2}^{2 \alpha}+b^{2 \alpha} r^{2 \alpha}\left(p_{2}^{\alpha} V+\gamma_{2}^{\alpha} \mu^{\alpha}\right)^{2}+2 b^{\alpha} p_{2}^{\alpha} \gamma_{2}^{\alpha}\left(p_{2}^{\alpha} r^{\alpha} V-\right.\right.\right.$ $\left.\left.\left.\left.\left.2 \sigma^{\alpha} p_{3}^{\alpha} \gamma_{2}^{\alpha}+r^{\alpha} \gamma_{2}^{\alpha} \mu^{\alpha}\right)\right)+d\left(-k_{T}^{\alpha} p_{2}^{\alpha}\left(-2+r^{\alpha}\right) V+r^{\alpha}\left(p_{2}^{\alpha}\left(-2+r^{\alpha}\right) \gamma_{2}^{\alpha}+b^{\alpha} r^{\alpha}\left(p_{2}^{\alpha} V+\gamma_{2}^{\alpha} \mu^{\alpha}\right)\right)\right)\right)\right)\right)$.

Then $E q_{2}$ is locally asymptotically stable.

Proof The Jacobian matrix of the model (2) at $E q_{2}$ is

$$
J\left(E q_{2}\right)=\left(\begin{array}{cccc}
\gamma_{1}^{\alpha}-k_{s}^{\alpha} \frac{V}{\gamma_{2}^{\alpha}} & 0 & 0 & 0 \\
0 & j_{22} & j_{23} & j_{24} \\
j_{31} & j_{32} & j_{33} & j_{34} \\
0 & 0 & 0 & -\gamma_{2}^{\alpha}
\end{array}\right),
$$

where

$$
\begin{gathered}
j_{22}=r^{\alpha}-\left(\frac{p_{2}^{\alpha} V\left(r^{\alpha} b^{\alpha}-k_{T}^{\alpha}\right)+r^{\alpha} \gamma_{2}^{\alpha}\left(p_{2}^{\alpha}-b^{\alpha} \mu^{\alpha}\right)-\sqrt{-4 \sigma^{\alpha} b^{\alpha} p_{2}^{\alpha} p_{3}^{\alpha} r^{\alpha} \gamma_{2}^{2 \alpha}+a^{2}}}{p_{2}^{\alpha} \gamma_{2}^{\alpha} r^{\alpha}}\right) \\
-\left(\frac{p_{2}^{\alpha} V\left(r^{\alpha} b^{\alpha}-k_{T}^{\alpha}\right)+r^{\alpha} \gamma_{2}^{\alpha}\left(p_{2}^{\alpha}+b^{\alpha} \mu^{\alpha}\right)+\sqrt{-4 \sigma^{\alpha} b^{\alpha} p_{2}^{\alpha} p_{3}^{\alpha} r^{\alpha} \gamma_{2}^{2 \alpha}+a^{2}}}{2 p_{2}^{\alpha} \gamma_{2}^{\alpha}}-k_{T}^{\alpha} \frac{V}{\gamma_{2}^{\alpha}}\right) . \\
j_{23}=-p_{3}^{\alpha} \frac{p_{2}^{\alpha} V\left(r^{\alpha} b^{\alpha}-k_{T}^{\alpha}\right)+r^{\alpha} \gamma_{2}^{\alpha}\left(p_{2}^{\alpha}-b^{\alpha} \mu^{\alpha}\right)-\sqrt{-4 \sigma^{\alpha} b^{\alpha} p_{2}^{\alpha} p_{3}^{\alpha} r^{\alpha} \gamma_{2}^{2 \alpha}+a^{2}}}{2 p_{2}^{\alpha} \gamma_{2}^{\alpha} r^{\alpha} b^{\alpha}} \\
j_{24}=-k_{T}^{\alpha} \frac{p_{2}^{\alpha} V^{\alpha}\left(r^{\alpha} b^{\alpha}-k_{T}^{\alpha}\right)+r^{\alpha} \gamma_{2}^{\alpha}\left(p_{2}^{\alpha}-b^{\alpha} \mu^{\alpha}\right)-\sqrt{-4 \sigma^{\alpha} b^{\alpha} p_{2}^{\alpha} p_{3}^{\alpha} r^{\alpha} \gamma_{2}^{2 \alpha}+a^{2}}}{2 p_{2}^{\alpha} \gamma_{2}^{\alpha} r^{\alpha} b^{\alpha}} . \\
j_{31}=p_{1}^{\alpha} \frac{p_{2}^{\alpha} V\left(r^{\alpha} b^{\alpha}-k_{T}^{\alpha}\right)+r^{\alpha} \gamma_{2}^{\alpha}\left(p_{2}^{\alpha}+b^{\alpha} \mu^{\alpha}\right)+\sqrt{-4 \sigma^{\alpha} b^{\alpha} p_{2}^{\alpha} p_{3}^{\alpha} r^{\alpha} \gamma_{2}^{2 \alpha}+a^{2}}}{2 p_{2}^{\alpha} p_{3}^{\alpha} \gamma_{2}^{\alpha}} . \\
j_{32}=j_{34}=-\frac{p_{2}^{\alpha} V\left(r^{\alpha} b^{\alpha}-k_{T}^{\alpha}\right)+r^{\alpha} \gamma_{2}^{\alpha}\left(p_{2}^{\alpha}+b^{\alpha} \mu^{\alpha}\right)+\sqrt{-4 \sigma^{\alpha} b^{\alpha} p_{2}^{\alpha} p_{3}^{\alpha} r^{\alpha} \gamma_{2}^{2 \alpha}+a^{2}}}{2 p_{3}^{\alpha} \gamma_{2}^{\alpha}} . \\
j_{33}=-\mu_{2}^{\alpha}-p_{2}^{\alpha}\left(\frac{p_{2}^{\alpha} V\left(r^{\alpha} b^{\alpha}-k_{T}^{\alpha}\right)+r^{\alpha} \gamma_{2}^{\alpha}\left(p_{2}^{\alpha}-b^{\alpha} \mu^{\alpha}\right)-\sqrt{-4 \sigma^{\alpha} b^{\alpha} p_{2}^{\alpha} p_{3}^{\alpha} r^{\alpha} \gamma_{2}^{2 \alpha}+a^{2}}}{V}\right) .
\end{gathered}
$$

The characteristic equation is $\left|J\left(E q_{2}\right)-\lambda I\right|=0$. Hence,

$$
\begin{aligned}
\left(\gamma_{1}^{\alpha}-k_{s}^{\alpha}\right. & \left.\frac{V}{\gamma_{2}^{\alpha}}-\lambda\right)\left(-\gamma_{2}^{\alpha}-\lambda\right)\left(\left(r^{\alpha}-\frac{p_{2}^{\alpha} V\left(r^{\alpha} b^{\alpha}-k_{T}^{\alpha}\right)+r^{\alpha} \gamma_{2}^{\alpha}\left(p_{2}^{\alpha}-b^{\alpha} \mu^{\alpha}\right)-\sqrt{-4 \sigma^{\alpha} b^{\alpha} p_{2}^{\alpha} p_{3}^{\alpha} r^{\alpha} \gamma_{2}^{2 \alpha}+a^{2}}}{p_{2}^{\alpha} \gamma_{2}^{\alpha} r^{\alpha}}\right.\right. \\
& \left.-\left(\frac{p_{2}^{\alpha} V\left(r^{\alpha} b^{\alpha}-k_{T}^{\alpha}\right)+r^{\alpha} \gamma_{2}^{\alpha}\left(p_{2}^{\alpha}+b^{\alpha} \mu^{\alpha}\right)+\sqrt{-4 \sigma^{\alpha} b^{\alpha} p_{2}^{\alpha} p_{3}^{\alpha} r^{\alpha} \gamma_{2}^{2 \alpha}+a^{2}}}{2 p_{2}^{\alpha} \gamma_{2}^{\alpha}}\right)-k_{T}^{\alpha} \frac{V}{\gamma_{2}^{\alpha}}-\lambda\right) \\
& \left(\left(-\mu^{\alpha}-p_{2}^{\alpha}\left(\frac{p_{2}^{\alpha} V\left(r^{\alpha} b^{\alpha}-k_{T}^{\alpha}\right)+r^{\alpha} \gamma_{2}^{\alpha}\left(p_{2}^{\alpha}-b^{\alpha} \mu^{\alpha}\right)-\sqrt{-4 \sigma^{\alpha} b^{\alpha} p_{2}^{\alpha} p_{3}^{\alpha} r^{\alpha} \gamma_{2}^{2 \alpha}+a^{2}}}{2 p_{2}^{\alpha} \gamma_{2}^{\alpha} r^{\alpha} b^{\alpha}}+\frac{V}{\gamma_{2}^{\alpha}}-\lambda\right)\right)\right. \\
& -\frac{p_{2}^{\alpha} V\left(r^{\alpha} b^{\alpha}-k_{T}^{\alpha}\right)+r^{\alpha} \gamma_{2}^{\alpha}\left(p_{2}^{\alpha}-b^{\alpha} \mu^{\alpha}\right)-\sqrt{-4 \sigma^{\alpha} b^{\alpha} p_{2}^{\alpha} p_{3}^{\alpha} r^{\alpha} \gamma_{2}^{2 \alpha}+a^{2}}}{2 \gamma_{2}^{\alpha} r^{\alpha} b^{\alpha}} \\
& \left.\left(\frac{p_{2}^{\alpha} V\left(r^{\alpha} b^{\alpha}-k_{T}^{\alpha}\right)+r^{\alpha} \gamma_{2}^{\alpha}\left(p_{2}^{\alpha}+b^{\alpha} \mu^{\alpha}\right)+\sqrt{-4 \sigma^{\alpha} b^{\alpha} p_{2}^{\alpha} p_{3}^{\alpha} r^{\alpha} \gamma_{2}^{2 \alpha}+a^{2}}}{2 p_{2}^{\alpha} \gamma_{2}^{\alpha}}\right)\right)=0 .
\end{aligned}
$$


Eigenvalues of $J\left(E q_{2}\right)$ are $\lambda_{1}=\gamma_{1}^{\alpha}-k_{s}^{\alpha} \frac{V}{\gamma_{2}^{\alpha}}, \lambda_{2}=-\gamma_{2}^{\alpha}$,

$$
\begin{aligned}
& \lambda_{3}=\frac{1}{\left(4 b^{\alpha} p_{2}^{\alpha} r^{2 \alpha} \gamma_{2}^{2 \alpha}\right)}\left(\left(2 b^{\alpha}+p_{2}^{\alpha}\right) r^{\alpha}\left(d+k_{T}^{\alpha} p_{2}^{\alpha} V\right) \gamma_{2}^{\alpha}-b^{\alpha} r^{3 \alpha} \gamma_{2}^{\alpha}\left(b^{\alpha} p_{2}^{\alpha} V-p_{2}^{\alpha} \gamma_{2}^{\alpha}+b^{\alpha} \gamma_{2}^{\alpha} \mu^{\alpha}\right)+\right. \\
& r^{2 \alpha} \gamma_{2}^{\alpha}\left(-p_{2}^{2 \alpha} \gamma_{2}^{\alpha}+2 b^{2 \alpha}\left(p_{2}^{\alpha} V+\gamma_{2}^{\alpha} \mu^{\alpha}\right)-b^{\alpha}\left(d+p_{2}^{\alpha}\left(\left(k_{T}^{\alpha}+p_{2}^{\alpha}\right) V+\gamma_{2}^{\alpha}\left(2+\mu^{\alpha}\right)\right)\right)\right) \\
& -\sqrt{ }\left(r ^ { 2 \alpha } \gamma _ { 2 } ^ { 2 \alpha } \left(\left(-p_{2}^{\alpha}\left(d+k_{T}^{\alpha} p_{2}^{\alpha} V-p_{2}^{\alpha} r^{\alpha} \gamma_{2}^{\alpha}\right)+b^{2 \alpha}\left(-2+r^{\alpha}\right) r^{\alpha}\left(p_{2}^{\alpha} V+\gamma_{2}^{\alpha} \mu^{\alpha}\right)+b^{\alpha}\left(d\left(-2+r^{\alpha}\right)\right.\right.\right.\right. \\
& \left.\left.-2 k_{T}^{\alpha} p_{2}^{\alpha} V+p_{2}^{\alpha} r^{\alpha}\left(\left(k_{T}^{\alpha}+p_{2}^{\alpha}\right) V+\gamma_{2}^{\alpha}\left(2-r^{\alpha}+\mu^{\alpha}\right)\right)\right)\right)^{2}-8 b^{\alpha} p_{2}^{\alpha}\left(-k_{T}^{2 \alpha} p_{2}^{2 \alpha}\left(-2+r^{\alpha}\right) V^{2}\right. \\
& -2 k_{T}^{\alpha} p_{2}^{\alpha} r^{\alpha} V\left(-p_{2}^{\alpha}\left(-2+r^{\alpha}\right) \gamma_{2}^{\alpha}+b^{\alpha}\left(p_{2}^{\alpha} V+\gamma_{2}^{\alpha} \mu^{\alpha}\right)\right) \\
& +r^{\alpha}\left(-p_{2}^{2 \alpha}\left(-2+r^{\alpha}\right) r^{\alpha} \gamma_{2}^{2 \alpha}+b^{2 \alpha} r^{2 \alpha}\left(p_{2}^{\alpha} V+\gamma_{2}^{\alpha} \mu^{\alpha}\right)^{2}+2 b^{\alpha} p_{2}^{\alpha} \gamma_{2}^{\alpha}\left(p_{2}^{\alpha} r^{\alpha} V-2 \sigma^{\alpha} p_{3}^{\alpha} \gamma_{2}^{\alpha}+r^{\alpha} \gamma_{2}^{\alpha} \mu^{\alpha}\right)\right) \\
& \left.\left.\left.\left.+d\left(-k_{T}^{\alpha} p_{2}^{\alpha}\left(-2+r^{\alpha}\right) V+r^{\alpha}\left(p_{2}^{\alpha}\left(-2+r^{\alpha}\right) \gamma_{2}^{\alpha}+b^{\alpha} r^{\alpha}\left(p_{2}^{\alpha} V+\gamma_{2}^{\alpha} \mu^{\alpha}\right)\right)\right)\right)\right)\right)\right) \text {. } \\
& \lambda_{4}=\frac{1}{\left(4 b^{\alpha} p_{2}^{\alpha} r^{2 \alpha} \gamma_{2}^{2 \alpha}\right)}\left(\left(2 b^{\alpha}+p_{2}^{\alpha}\right) r^{\alpha}\left(d+k_{T}^{\alpha} p_{2}^{\alpha} V\right) \gamma_{2}^{\alpha}-b^{\alpha} r^{3 \alpha} \gamma_{2}^{\alpha}\left(b^{\alpha} p_{2}^{\alpha} V-p_{2}^{\alpha} \gamma_{2}^{\alpha}+b^{\alpha} \gamma_{2}^{\alpha} \mu^{\alpha}\right)\right. \\
& +r^{2 \alpha} \gamma_{2}^{\alpha}\left(-p_{2}^{2 \alpha} \gamma_{2}^{\alpha}+2 b^{2 \alpha}\left(p_{2}^{\alpha} V+\gamma_{2}^{\alpha} \mu^{\alpha}\right)-b^{\alpha}\left(d+p_{2}^{\alpha}\left(\left(k_{T}^{\alpha}+p_{2}^{\alpha}\right) V+\gamma_{2}^{\alpha}\left(2+\mu^{\alpha}\right)\right)\right)\right) \\
& +\sqrt{ }\left(r ^ { 2 \alpha } \gamma _ { 2 } ^ { 2 \alpha } \left(\left(-p_{2}^{\alpha}\left(d+k_{T}^{\alpha} p_{2}^{\alpha} V-p_{2}^{\alpha} r^{\alpha} \gamma_{2}^{\alpha}\right)+b^{2 \alpha}\left(-2+r^{\alpha}\right) r^{\alpha}\left(p_{2}^{\alpha} V+\gamma_{2}^{\alpha} \mu^{\alpha}\right)\right.\right.\right. \\
& \left.+b^{\alpha}\left(d\left(-2+r^{\alpha}\right)-2 k_{T}^{\alpha} p_{2}^{\alpha} V+p_{2}^{\alpha} r^{\alpha}\left(\left(k_{T}^{\alpha}+p_{2}^{\alpha}\right) V+\gamma_{2}^{\alpha}\left(2-r^{\alpha}+\mu^{\alpha}\right)\right)\right)\right)^{2} \\
& -8 b^{\alpha} p_{2}^{\alpha}\left(-k_{T}^{2 \alpha} p_{2}^{2 \alpha}\left(-2+r^{\alpha}\right) V^{2}-2 k_{T}^{\alpha} p_{2}^{\alpha} r^{\alpha} V^{\alpha}\left(-p_{2}^{\alpha}\left(-2+r^{\alpha}\right) \gamma_{2}^{\alpha}+b^{\alpha}\left(p_{2}^{\alpha} V+\gamma_{2}^{\alpha} \mu^{\alpha}\right)\right)+r^{\alpha}\right. \\
& \left(-p_{2}^{2 \alpha}\left(-2+r^{\alpha}\right) r^{\alpha} \gamma_{2}^{2 \alpha}+b^{2 \alpha} r^{2 \alpha}\left(p_{2}^{\alpha} V+\gamma_{2}^{\alpha} \mu^{\alpha}\right)^{2}+2 b^{\alpha} p_{2}^{\alpha} \gamma_{2}^{\alpha}\left(p_{2}^{\alpha} r^{\alpha} V-2 \sigma^{\alpha} p_{3}^{\alpha} \gamma_{2}^{\alpha}+r^{\alpha} \gamma_{2}^{\alpha} \mu^{\alpha}\right)\right) \\
& \left.\left.\left.\left.+d\left(-k_{T}^{\alpha} p_{2}^{\alpha}\left(-2+r^{\alpha}\right) V+r^{\alpha}\left(p_{2}^{\alpha}\left(-2+r^{\alpha}\right) \gamma_{2}^{\alpha}+b^{\alpha} r^{\alpha}\left(p_{2}^{\alpha} V+\gamma_{2}^{\alpha} \mu^{\alpha}\right)\right)\right)\right)\right)\right)\right) \text {. }
\end{aligned}
$$

From the conditions we have $\lambda_{i}<0$ for $i=1,2,3,4$. Therefore, $\left|\arg \left(\lambda_{i}\right)\right|>\frac{\alpha \pi}{2}$ by Theorem (1) and $E q_{2}$ is locally asymptotically stable.

Theorem 5 Let $E q_{3} \in D_{1}$ be the equilibrium point of system (2) and $\left(p_{2}^{\alpha}, p_{3}^{\alpha}, \mu^{\alpha}, \gamma_{2}^{\alpha}, \sigma^{\alpha}, r^{\alpha}, b^{\alpha}, V, k_{T}^{\alpha}\right) \in D_{1} \cap\left(Q^{*} \cup P^{*}\right)$, where $Q^{*}=$ $\left\{\left(p_{2}^{\alpha}, p_{3}^{\alpha}, \mu^{\alpha}, \gamma_{2}^{\alpha}, \sigma^{\alpha}, r^{\alpha}, b^{\alpha}, V, k_{T}^{\alpha}\right) \in R_{+}^{9}: d^{2}, d 1 \geq 0, \gamma_{1}^{\alpha} \gamma_{2}^{\alpha}<k_{S}^{\alpha} V\right.$ and $\left(\left(2 b^{\alpha}+p_{2}^{\alpha}\right)\left(-d+k_{T}^{\alpha} p_{2}^{\alpha} V\right) r^{\alpha} \gamma_{2}^{\alpha}-b^{\alpha} r^{3 \alpha} \gamma_{2}^{\alpha}\left(b^{\alpha} p_{2}^{\alpha} V-p_{2}^{\alpha} \gamma_{2}^{\alpha}+\right.\right.$ $\left.\left.\left.b^{\alpha} \gamma_{2}^{\alpha} \mu^{\alpha}\right)+r^{2 \alpha} \gamma_{2}^{\alpha}\left(-p_{2}^{2 \alpha} \gamma_{2}^{\alpha}+2 b^{2 \alpha}\left(p_{2}^{\alpha} V+\gamma_{2}^{\alpha} \mu^{\alpha}\right)-b^{\alpha}\left(-d+p_{2}^{\alpha}\left(\left(k_{T}^{\alpha}+p_{2}^{\alpha}\right) V+\gamma_{2}^{\alpha}\left(2+\mu^{\alpha}\right)\right)\right)\right)-\sqrt{d_{1}}\right)<0\right\}$.

$P^{*}=\left\{\left(p_{2}^{\alpha}, p_{3}^{\alpha}, \mu^{\alpha}, \gamma_{2}^{\alpha}, \sigma^{\alpha}, r^{\alpha}, b^{\alpha}, V, k_{T}^{\alpha}\right) \in R_{+}^{9}: d^{2}\right.$ or $d 1<0, \gamma_{1}^{\alpha} \gamma_{2}^{\alpha}<k_{5}^{\alpha} V$ and $\operatorname{Re}\left(\left(\left(2 b^{\alpha}+p_{2}^{\alpha}\right)\left(-d+k_{T}^{\alpha} p_{2}^{\alpha} V\right) r^{\alpha} \gamma_{2}^{\alpha}-b^{\alpha} r^{3 \alpha} \gamma_{2}^{\alpha}\left(b^{\alpha} p_{2}^{\alpha} V-\right.\right.\right.$ $\left.\left.\left.\left.p_{2}^{\alpha} \gamma_{2}^{\alpha}+b^{\alpha} \gamma_{2}^{\alpha} \mu^{\alpha}\right)+r^{2 \alpha} \gamma_{2}^{\alpha}\left(-p_{2}^{2 \alpha} \gamma_{2}^{\alpha}+2 b^{2 \alpha}\left(p_{2}^{\alpha} V+\gamma_{2}^{\alpha} \mu^{\alpha}\right)-b^{\alpha}\left(-d+p_{2}^{\alpha}\left(\left(k_{T}^{\alpha}+p_{2}^{\alpha}\right) V+\gamma_{2}^{\alpha}\left(2+\mu^{\alpha}\right)\right)\right)\right)+\sqrt{d_{1}}\right)\right)<0\right\}$,

where $d=\sqrt{-4 \sigma^{\alpha} b^{\alpha} p_{2}^{\alpha} p_{3}^{\alpha} r^{\alpha} \gamma_{2}^{2 \alpha}+\left(k_{T}^{\alpha} p_{2}^{\alpha} V-b^{\alpha} p_{2}^{\alpha} r^{\alpha} V-p_{2}^{\alpha} r^{\alpha} \gamma_{2}^{\alpha}-b^{\alpha} r^{\alpha} \gamma_{2}^{\alpha} \mu^{\alpha}\right)^{2}}$ and $d_{1}=\left(r^{2 \alpha} \gamma_{2}^{2 \alpha}\left(\left(-p_{2}^{\alpha}\left(d+k_{T}^{\alpha} p_{2}^{\alpha} V-p_{2}^{\alpha} r^{\alpha} \gamma_{2}^{\alpha}\right)+\right.\right.\right.$ $\left.b^{2 \alpha}\left(-2+r^{\alpha}\right) r^{\alpha}\left(p_{2}^{\alpha} V+\gamma_{2}^{\alpha} \mu^{\alpha}\right)+b^{\alpha}\left(d\left(-2+r^{\alpha}\right)-2 k_{T}^{\alpha} p_{2}^{\alpha} V+p_{2}^{\alpha} r^{\alpha}\left(\left(k_{T}^{\alpha}+p_{2}^{\alpha}\right) V+\gamma_{2}^{\alpha}\left(2-r^{\alpha}+\mu^{\alpha}\right)\right)\right)\right)^{2}-8 b^{\alpha} p_{2}^{\alpha}$

$\left(-k_{T}^{2 \alpha} p_{2}^{2 \alpha}\left(-2+r^{\alpha}\right) V^{2}-2 k_{T}^{\alpha} p_{2}^{\alpha} r^{\alpha} V\left(-p_{2}^{\alpha}\left(-2+r^{\alpha}\right) \gamma_{2}^{\alpha}+b^{\alpha}\left(p_{2}^{\alpha} V+\gamma_{2}^{\alpha} \mu^{\alpha}\right)\right)+r^{\alpha}\left(-p_{2}^{2 \alpha}\left(-2+r^{\alpha}\right) r^{\alpha} \gamma_{2}^{2 \alpha}+b^{2 \alpha} r^{2 \alpha}\left(p_{2}^{\alpha} V+\gamma_{2}^{\alpha} \mu^{\alpha}\right)^{2}+2 b^{\alpha} p_{2}^{\alpha} \gamma_{2}^{\alpha}\left(p_{2}^{\alpha} r V-\right.\right.\right.$ $\left.\left.\left.\left.\left.2 \sigma^{\alpha} p_{3}^{\alpha} \gamma_{2}^{\alpha}+r^{\alpha} \gamma_{2}^{\alpha} \mu^{\alpha}\right)\right)+d\left(-k_{T}^{\alpha} p_{2}^{\alpha}\left(-2+r^{\alpha}\right) V+r^{\alpha}\left(p_{2}^{\alpha}\left(-2+r^{\alpha}\right) \gamma_{2}^{\alpha}+b^{\alpha} r^{\alpha}\left(p_{2}^{\alpha} V+\gamma_{2}^{\alpha} \mu^{\alpha}\right)\right)\right)\right)\right)\right)$.

Then $E q_{3}$ is locally asymptotically stable.

Proof The Jacobian matrix of the model (2) at $E q_{3}$ is

$$
J\left(E q_{3}\right)=\left(\begin{array}{cccc}
\gamma_{1}^{\alpha}-k_{s}^{\alpha} \frac{V}{\gamma_{2}^{\alpha}} & 0 & 0 & 0 \\
0 & j_{22}^{*} & j_{23}^{*} & j_{24} \\
j_{31}^{*} & j_{32}^{*} & j_{33}^{*} & j_{34}^{*} \\
0 & 0 & 0 & -\gamma_{2}^{\alpha}
\end{array}\right),
$$

where

$$
\begin{aligned}
j_{22}^{*}= & r^{\alpha}-\left(\frac{p_{2}^{\alpha} V\left(r^{\alpha} b^{\alpha}-k_{T}^{\alpha}\right)+r^{\alpha} \gamma_{2}^{\alpha}\left(p_{2}^{\alpha}-b^{\alpha} \mu^{\alpha}\right)+\sqrt{-4 \sigma^{\alpha} b^{\alpha} p_{2}^{\alpha} p_{3}^{\alpha} r^{\alpha} \gamma_{2}^{2 \alpha}+a^{2}}}{p_{2}^{\alpha} \gamma_{2}^{\alpha} r^{\alpha}}\right) \\
& -\left(\frac{p_{2}^{\alpha} V\left(r^{\alpha} b^{\alpha}-k_{T}^{\alpha}\right)+r^{\alpha} \gamma_{2}^{\alpha}\left(p_{2}^{\alpha}+b^{\alpha} \mu^{\alpha}\right)-\sqrt{-4 \sigma^{\alpha} b^{\alpha} p_{2}^{\alpha} p_{3}^{\alpha} r^{\alpha} \gamma_{2}^{2 \alpha}+a^{2}}}{2 p_{2}^{\alpha} \gamma_{2}^{\alpha}}-k_{T}^{\alpha} \frac{V}{\gamma_{2}^{\alpha}}\right) . \\
j_{23}^{*}= & -p_{3}^{\alpha} \frac{p_{2}^{\alpha} V\left(r b^{\alpha}-k_{T}^{\alpha}\right)+r^{\alpha} \gamma_{2}^{\alpha}\left(p_{2}^{\alpha}-b^{\alpha} \mu^{\alpha}\right)+\sqrt{-4 \sigma^{\alpha} b^{\alpha} p_{2}^{\alpha} p_{3}^{\alpha} r^{\alpha} \gamma_{2}^{2 \alpha}+a^{2}}}{2 p_{2}^{\alpha} \gamma_{2}^{\alpha} r^{\alpha} b^{\alpha}} . \\
j_{24}^{*}= & -k_{T}^{\alpha} \frac{p_{2}^{\alpha} V\left(r^{\alpha} b^{\alpha}-k_{T}^{\alpha}\right)+r^{\alpha} \gamma_{2}^{\alpha}\left(p_{2}^{\alpha}-b^{\alpha} \mu^{\alpha}\right)+\sqrt{-4 \sigma^{\alpha} b^{\alpha} p_{2}^{\alpha} p_{3}^{\alpha} r^{\alpha} \gamma_{2}^{2 \alpha}+a^{2}}}{2 p_{2}^{\alpha} \gamma_{2}^{\alpha} r^{\alpha} b^{\alpha}} . \\
j_{31}^{*}= & p_{1}^{\alpha} \frac{p_{2}^{\alpha} V\left(r^{\alpha} b^{\alpha}-k_{T}^{\alpha}\right)+r^{\alpha} \gamma_{2}^{\alpha}\left(p_{2}^{\alpha}+b^{\alpha} \mu^{\alpha}\right)-\sqrt{-4 \sigma^{\alpha} b^{\alpha} p_{2}^{\alpha} p_{3}^{\alpha} r^{\alpha} \gamma_{2}^{2}+a^{2}}}{2 p_{2}^{\alpha} p_{3}^{\alpha} \gamma_{2}^{\alpha}} . \\
j_{32}^{*}= & j_{34}^{*}=-\frac{p_{2}^{\alpha} V\left(r^{\alpha} b^{\alpha}-k_{T}^{\alpha}\right)+r^{\alpha} \gamma_{2}^{\alpha}\left(p_{2}^{\alpha}+b^{\alpha} \mu^{\alpha}\right)-\sqrt{-4 \sigma^{\alpha} b^{\alpha} p_{2}^{\alpha} p_{3}^{\alpha} r^{\alpha} \gamma_{2}^{2 \alpha}+a^{2}}}{2 p_{3}^{\alpha} \gamma_{2}^{\alpha}} . \\
j_{33}^{*}= & -\mu^{\alpha}-p_{2}^{\alpha}\left(\frac{p_{2}^{\alpha} V\left(r^{\alpha} b^{\alpha}-k_{T}^{\alpha}\right)+r^{\alpha} \gamma_{2}^{\alpha}\left(p_{2}^{\alpha}-b^{\alpha} \mu^{\alpha}\right)+\sqrt{-4 \sigma^{\alpha} b^{\alpha} p_{2}^{\alpha} p_{3}^{\alpha} r^{\alpha} \gamma_{2}^{2 \alpha}+a^{2}}}{2 p_{2}^{\alpha} \gamma_{2}^{\alpha} r^{\alpha} b^{\alpha}}+\frac{V}{\gamma_{2}^{\alpha}}\right) .
\end{aligned}
$$


The characteristic equation is $\left|J\left(E q_{3}\right)-\lambda I\right|=0$. Hence,

$$
\begin{gathered}
\left(\gamma_{1}^{\alpha}-k_{s}^{\alpha} \frac{V}{\gamma_{2}^{\alpha}}-\lambda\right)\left(-\gamma_{2}^{\alpha}-\lambda\right)\left(\left(r^{\alpha}-\frac{p_{2}^{\alpha} V\left(r^{\alpha} b^{\alpha}-k_{T}^{\alpha}\right)+r^{\alpha} \gamma_{2}^{\alpha}\left(p_{2}^{\alpha}-b^{\alpha} \mu^{\alpha}\right)+\sqrt{-4 \sigma^{\alpha} b^{\alpha} p_{2}^{\alpha} p_{3}^{\alpha} r^{\alpha} \gamma_{2}^{2 \alpha}+a^{2}}}{p_{2}^{\alpha} \gamma_{2}^{\alpha} r^{\alpha}}\right.\right. \\
\left.-\left(\frac{p_{2}^{\alpha} V\left(r^{\alpha} b^{\alpha}-k_{T}^{\alpha}\right)+r^{\alpha} \gamma_{2}^{\alpha}\left(p_{2}^{\alpha}+b^{\alpha} \mu^{\alpha}\right)-\sqrt{-4 \sigma^{\alpha} b^{\alpha} p_{2}^{\alpha} p_{3}^{\alpha} r^{\alpha} \gamma_{2}^{2 \alpha}+a^{2}}}{2 p_{2}^{\alpha} \gamma_{2}^{\alpha}}\right)-k_{T}^{\alpha} \frac{V}{\gamma_{2}^{\alpha}}-\lambda\right) \\
\left(\left(-\mu^{\alpha}-\left(\frac{p_{2}^{\alpha} V\left(r^{\alpha} b^{\alpha}-k_{T}^{\alpha}\right)+r^{\alpha} \gamma_{2}^{\alpha}\left(p_{2}^{\alpha}-b^{\alpha} \mu^{\alpha}\right)+\sqrt{-4 \sigma^{\alpha} b^{\alpha} p_{2}^{\alpha} p_{3}^{\alpha} r^{\alpha} \gamma_{2}^{2 \alpha}+a^{2}}}{2 \gamma_{2}^{\alpha} r^{\alpha} b^{\alpha}}+\frac{V}{\gamma_{2}^{\alpha}}-\lambda\right)\right)\right. \\
-\frac{p_{2}^{\alpha} V\left(r^{\alpha} b^{\alpha}-k_{T}^{\alpha}\right)+r^{\alpha} \gamma_{2}^{\alpha}\left(p_{2}^{\alpha}-b^{\alpha} \mu^{\alpha}\right)+\sqrt{-4 \sigma^{\alpha} b^{\alpha} p_{2}^{\alpha} p_{3}^{\alpha} r^{\alpha} \gamma_{2}^{2 \alpha}+a^{2}}}{2 \gamma_{2}^{\alpha} r^{\alpha} b^{\alpha}} \\
\left.\left(\frac{p_{2}^{\alpha} V\left(r^{\alpha} b^{\alpha}-k_{T}^{\alpha}\right)+r^{\alpha} \gamma_{2}^{\alpha}\left(p_{2}^{\alpha}+b^{\alpha} \mu^{\alpha}\right)-\sqrt{-4 \sigma^{\alpha} b^{\alpha} p_{2}^{\alpha} p_{3}^{\alpha} r^{\alpha} \gamma_{2}^{2 \alpha}+a^{2}}}{2 p_{2}^{\alpha} \gamma_{2}^{\alpha}}\right)\right)=0 .
\end{gathered}
$$

Eigenvalues of $J\left(E q_{3}\right)$ are $\lambda_{1}=\gamma_{1}^{\alpha}-k_{s}^{\alpha} \frac{V}{\gamma_{2}^{\alpha}}, \lambda_{2}=-\gamma_{2}^{\alpha}$,

$$
\begin{aligned}
& \lambda_{3}=\frac{1}{\left(4 b^{\alpha} p_{2}^{\alpha} r^{2 \alpha} \gamma_{2}^{2 \alpha}\right)}\left(\left(2 b^{\alpha}+p_{2}^{\alpha}\right) r^{\alpha}\left(-d+k_{T}^{\alpha} p_{2}^{\alpha} V\right) \gamma_{2}^{\alpha}-b^{\alpha} r^{3 \alpha} \gamma_{2}^{\alpha}\left(b^{\alpha} p_{2}^{\alpha} V-p_{2}^{\alpha} \gamma_{2}^{\alpha}+b^{\alpha} \gamma_{2}^{\alpha} \mu^{\alpha}\right)\right. \\
& +r^{2 \alpha} \gamma_{2}^{\alpha}\left(-p_{2}^{2 \alpha} \gamma_{2}^{\alpha}+2 b^{2 \alpha}\left(p_{2}^{\alpha} V+\gamma_{2}^{\alpha} \mu^{\alpha}\right)-b^{\alpha}\left(d+p_{2}^{\alpha}\left(\left(k_{T}^{\alpha}+p_{2}^{\alpha}\right) V+\gamma_{2}^{\alpha}\left(2+\mu^{\alpha}\right)\right)\right)\right) \\
& -\left(r ^ { 2 \alpha } \gamma _ { 2 } ^ { 2 \alpha } \left(\left(-p_{2}^{\alpha}\left(-d+k_{T}^{\alpha} p_{2}^{\alpha} V-p_{2}^{\alpha} r^{\alpha} \gamma_{2}^{\alpha}\right)+b^{2 \alpha}\left(-2+r^{\alpha}\right) r^{\alpha}\left(p_{2}^{\alpha} V+\gamma_{2}^{\alpha} \mu^{\alpha}\right)\right.\right.\right. \\
& \left.+b^{\alpha}\left(-d\left(-2+r^{\alpha}\right)-2 k_{T}^{\alpha} p_{2}^{\alpha} V+p_{2}^{\alpha} r^{\alpha}\left(\left(k_{T}^{\alpha}+p_{2}^{\alpha}\right) V+\gamma_{2}^{\alpha}\left(2-r^{\alpha}+\mu^{\alpha}\right)\right)\right)\right)^{2} \\
& -8 b^{\alpha} p_{2}^{\alpha}\left(-k_{T}^{2 \alpha} p_{2}^{2 \alpha}\left(-2+r^{\alpha}\right) V^{2}-2 k_{T}^{\alpha} p_{2}^{\alpha} r^{\alpha} V\left(-p_{2}^{\alpha}\left(-2+r^{\alpha}\right) \gamma_{2}^{\alpha}+b^{\alpha}\left(p_{2}^{\alpha} V+\gamma_{2}^{\alpha} \mu^{\alpha}\right)\right)+r^{\alpha}\left(-p_{2}^{2 \alpha}\left(-2+r^{\alpha}\right) r^{\alpha} \gamma_{2}^{2 \alpha}+b^{2 \alpha} r^{2 \alpha}\left(p_{2}^{\alpha} V\right.\right.\right. \\
& \left.\left.\left.\left.\left.\left.+\gamma_{2}^{\alpha} \mu^{\alpha}\right)^{2}+2 b^{\alpha} p_{2}^{\alpha} \gamma_{2}^{\alpha}\left(p_{2}^{\alpha} r^{\alpha} V-2 \sigma^{\alpha} p_{3}^{\alpha} \gamma_{2}^{\alpha}+r^{\alpha} \gamma_{2}^{\alpha} \mu^{\alpha}\right)\right)-d\left(-k_{T}^{\alpha} p_{2}^{\alpha}\left(-2+r^{\alpha}\right) V+r^{\alpha}\left(p_{2}^{\alpha}\left(-2+r^{\alpha}\right) \gamma_{2}^{\alpha}+b^{\alpha} r^{\alpha}\left(p_{2}^{\alpha} V+\gamma_{2}^{\alpha} \mu^{\alpha}\right)\right)\right)\right)\right)\right)^{1 / 2}\right) \text {, } \\
& \lambda_{4}=\frac{1}{\left(4 b^{\alpha} p_{2}^{\alpha} r^{2 \alpha} \gamma_{2}^{2 \alpha}\right)}\left(\left(2 b^{\alpha}+p_{2}^{\alpha}\right) r^{\alpha}\left(-d+k_{T}^{\alpha} p_{2}^{\alpha} V\right) \gamma_{2}^{\alpha}-b^{\alpha} r^{3 \alpha} \gamma_{2}^{\alpha}\left(b^{\alpha} p_{2}^{\alpha} V-p_{2}^{\alpha} \gamma_{2}^{\alpha}+b^{\alpha} \gamma_{2}^{\alpha} \mu^{\alpha}\right)\right. \\
& +r^{2 \alpha} \gamma_{2}^{\alpha}\left(-p_{2}^{2 \alpha} \gamma_{2}^{\alpha}+2 b^{2 \alpha}\left(p_{2}^{\alpha} V+\gamma_{2}^{\alpha} \mu^{\alpha}\right)-b^{\alpha}\left(-d+p_{2}^{\alpha}\left(\left(k_{T}^{\alpha}+p_{2}^{\alpha}\right) V+\gamma_{2}^{\alpha}\left(2+\mu^{\alpha}\right)\right)\right)\right) \\
& +\left(r ^ { 2 \alpha } \gamma _ { 2 } ^ { 2 \alpha } \left(\left(-p_{2}^{\alpha}\left(d+k_{T}^{\alpha} p_{2}^{\alpha} V-p_{2}^{\alpha} r^{\alpha} \gamma_{2}^{\alpha}\right)+b^{2 \alpha}\left(-2+r^{\alpha}\right) r^{\alpha}\left(p_{2}^{\alpha} V+\gamma_{2}^{\alpha} \mu^{\alpha}\right)+b^{\alpha}\left(d^{\alpha}\left(-2+r^{\alpha}\right)-2 k_{T}^{\alpha} p_{2}^{\alpha} V+p_{2}^{\alpha} r^{\alpha}\left(\left(k_{T}^{\alpha}+p_{2}^{\alpha}\right) V+\right.\right.\right.\right.\right. \\
& \left.\left.\left.\gamma_{2}^{\alpha}\left(2-r^{\alpha}+\mu^{\alpha}\right)\right)\right)\right)^{2}-8 b^{\alpha} p_{2}^{\alpha}\left(-k_{T}^{2 \alpha} p_{2}^{2 \alpha}\left(-2+r^{\alpha}\right) V^{2}-2 k_{T}^{\alpha} p_{2}^{\alpha} r^{\alpha} V^{\alpha}\left(-p_{2}^{\alpha}\left(-2+r^{\alpha}\right) \gamma_{2}^{\alpha}+b^{\alpha}\left(p_{2}^{\alpha} V+\gamma_{2}^{\alpha} \mu^{\alpha}\right)\right)\right. \\
& +r^{\alpha}\left(-p_{2}^{2 \alpha}\left(-2+r^{\alpha}\right) r^{\alpha} \gamma_{2}^{2 \alpha}+b^{2 \alpha} r^{2 \alpha}\left(p_{2}^{\alpha} V+\gamma_{2}^{\alpha} \mu^{\alpha}\right)^{2}+2 b^{\alpha} p_{2}^{\alpha} \gamma_{2}^{\alpha}\left(p_{2}^{\alpha} r^{\alpha} V-2 \sigma^{\alpha} p_{3}^{\alpha} \gamma_{2}^{\alpha}\right.\right. \\
& \left.\left.\left.\left.\left.\left.+r^{\alpha} \gamma_{2}^{\alpha} \mu^{\alpha}\right)\right)-d\left(-k_{T}^{\alpha} p_{2}^{\alpha}\left(-2+r^{\alpha}\right) V+r^{\alpha}\left(p_{2}^{\alpha}\left(-2+r^{\alpha}\right) \gamma_{2}^{\alpha}+b^{\alpha} r^{\alpha}\left(p_{2}^{\alpha} V+\gamma_{2}^{\alpha} \mu^{\alpha}\right)\right)\right)\right)\right)\right)^{1 / 2}\right) \text {. }
\end{aligned}
$$

From the conditions we have $\lambda_{i}<$ o for $i=1,2,3,4$. Therefore, $\left|\arg \left(\lambda_{i}\right)\right|>\frac{\alpha \pi}{2}$, and by Theorem (1), $E q_{3}$ is locally asymptotically stable.

\section{A numerical technique for the proposed fractional-order model}

In this section, numerical solution of system (3) is carried out using the Predictor-Corrector method of Adams-Bashforth-Moulton [32, 33] for different $\alpha \in(0,1]$. We implement the Caputo fractional operator to provide the numerical simulation of a nonlinear fractional order system. The following Cauchy-type ODE is taken into account with respect to the Caputo operator of order $\alpha$ :

$$
{ }_{C} D_{t}^{\alpha} D_{t}^{\alpha} \Phi(t)=f(t, \Phi(t)), \quad \Phi^{(k)}(0)=\Phi_{0}^{k}, \quad 0<\alpha \leq 1, \quad 0<t \leq \tau,
$$

where $k=0,1, \ldots, n-1$, and $n=\lceil\alpha\rceil$. Equation (7) is equivalent to the following Volterra equation:

$$
\Phi(t)=\sum_{k=0}^{n-1} \Phi_{0}^{(k)} \frac{t^{k}}{k !}+\frac{1}{\Gamma(\alpha)} \int_{0}^{t}(t-s)^{\alpha-1} f(s, \Phi(s)) d s .
$$

By considering this proposed predictor-corrector scheme associated with the Adam-Bashforth-Moulton algorithm $[4,6]$ to have the numerical solutions of the proposed model, we can take $h=\tau / N, t_{z}=z h$, and $z=0,1, \ldots, N \in \mathrm{Z}^{+}$, by letting $\Phi_{z} \approx \Phi\left(t_{z}\right)$, it can be discretized as follows, i.e., the corresponding corrector formula [6]

$$
\begin{aligned}
S_{q+1}=\sum_{z=0}^{q-1} S_{0}^{(z)} \frac{t_{q+1}^{z}}{z !}+ & \frac{h^{\alpha}}{\Gamma(\alpha+2)} \sum_{z=0}^{q}\left(p_{z, q+1}\right)\left(\gamma_{1}^{\alpha} S_{z}-k_{s}^{\alpha} M_{z} S_{z}\right)+\frac{h^{\alpha}}{\Gamma(\alpha+2)} \sum_{z=0}^{q}\left(p_{q+1, q+1}\right)\left(\gamma_{1}^{\alpha} S_{q+1}^{P F}-k_{s}^{\alpha} M_{q+1}^{P F} S_{q+1}^{P F}\right) \\
T_{q+1}= & \sum_{z=0}^{q-1} T_{0}^{(z)} \frac{t_{q+1}^{z}}{z !}+\frac{h^{\alpha}}{\Gamma(\alpha+2)} \sum_{z=0}^{q}\left(p_{z, q+1}\right)\left(r^{\alpha}\left(1-b^{\alpha} T_{z}\right) T_{z}-\left(p_{3}^{\alpha} E_{z}+k_{t}^{\alpha} M_{z}\right) T_{z}\right) \\
& +\frac{h^{\alpha}}{\Gamma(\alpha+2)} \sum_{z=0}^{q}\left(p_{q+1, q+1}\right)\left(r^{\alpha}\left(1-b^{\alpha} T_{q+1}^{P F}\right) T_{q+1}^{P F}-\left(p_{3}^{\alpha} E_{q+1}^{P F}+k_{t}^{\alpha} M_{q+1}^{P F}\right) T_{q+1}^{P F}\right)
\end{aligned}
$$




$$
\begin{aligned}
E_{q+1}= & \sum_{z=0}^{q-1} E_{0}^{(z)} \frac{t_{q+1}^{z}}{z !}+\frac{h^{\alpha}}{\Gamma(\alpha+2)} \sum_{z=0}^{q}\left(p_{z, q+1}\right)\left(a^{\alpha}-\mu^{\alpha} E_{z}+\frac{p_{1}^{\alpha} E_{z} S_{z}}{S_{z}+1}-p_{2}^{\alpha}\left(T_{z}+M_{z}\right) E_{z}\right) \\
& +\frac{h^{\alpha}}{\Gamma(\alpha+2)} \sum_{z=0}^{q}\left(p_{q+1, q+1}\right)\left(a^{\alpha}-\mu^{\alpha} E_{q+1}^{P F}+\frac{p_{1}^{\alpha} E_{q+1}^{P F} S_{q+1}^{P F}}{S_{q+1}^{P F}+1}-p_{2}^{\alpha}\left(T_{q+1}^{P F}+M_{q+1}^{P F}\right) E_{q+1}^{P F}\right), \\
M_{q+1}= & \sum_{z=0}^{q-1} M_{0}^{(z)} \frac{t_{q+1}^{z}}{z !}+\frac{h^{\alpha}}{\Gamma(\alpha+2)} \sum_{z=0}^{q}\left(p_{z, q+1}\right)\left(-\gamma_{2}^{\alpha} M_{z}+V(t)\right)+\frac{h^{\alpha}}{\Gamma(\alpha+2)} \sum_{z=0}^{q}\left(p_{q+1, q+1}\right)\left(-\gamma_{2}^{\alpha} M_{q+1}^{P F}+V(t)\right),
\end{aligned}
$$

where

$$
p_{z, q+1}= \begin{cases}q^{\alpha+1}-(q-\alpha)(q+1)^{\alpha}, & \text { if } z=0 \\ (q-z+2)^{\alpha+1}+(q-z)^{\alpha+1}-2(q-z+1)^{\alpha+1}, & \text { if } 1 \leq z \leq q \\ 1, & \text { if } z=q+1\end{cases}
$$

Subsequently, the following step is to construct the coincident predictor formula $\Phi_{q+1}^{P F}$. One can compute the proposed predictor formula as

$$
\begin{aligned}
S_{q+1}^{P F} & =\sum_{z=0}^{q-1} S_{0}^{(z)} \frac{t_{q+1}^{z}}{z !}+\frac{h^{\alpha}}{\Gamma(\alpha+1)} \sum_{z=0}^{q}\left(j_{z, \alpha} q_{+1}\right)\left(\gamma_{1}^{\alpha} S_{z}-k_{s}^{\alpha} M_{z} S_{z}\right) \\
T_{q+1}^{P F} & =\sum_{z=0}^{q-1} T_{0}^{(z)} \frac{t_{q+1}^{z}}{z !}+\frac{h^{\alpha}}{\Gamma(\alpha+1)} \sum_{z=0}^{q}\left(j_{z, q+1}\right)\left(r^{\alpha}\left(1-b^{\alpha} T_{z}\right) T_{z}-\left(p_{3}^{\alpha} E_{z}+k_{t}^{\alpha} M_{z}\right) T_{z}\right) \\
E_{q+1}^{P F} & =\sum_{z=0}^{q-1} E_{0}^{(z)} \frac{t_{q+1}^{z}}{z !}+\frac{h^{\alpha}}{\Gamma(\alpha+1)} \sum_{z=0}^{q}\left(j_{z, q+1}\right)\left(a^{\alpha}-\mu^{\alpha} E_{z}+\frac{p_{1}^{\alpha} E_{z} S_{z}}{S_{z}+1}-p_{2}^{\alpha}\left(T_{z}+M_{z}\right) E_{z}\right) \\
M_{q+1}^{P F} & =\sum_{z=0}^{q-1} M_{0}^{(z)} \frac{t_{q+1}^{z}}{z !}+\frac{h^{\alpha}}{\Gamma(\alpha+1)} \sum_{z=0}^{q}\left(j_{z, q+1}\right)\left(-\gamma_{2}^{\alpha} M_{z}+V(t)\right)
\end{aligned}
$$

where

$$
j_{z, q+1}=(q+1-z)^{\alpha}-(q-z)^{\alpha}
$$

\section{Memory trace and hereditary traits}

To examine the behaviour of the proposed model (2), we use the Caputo operator defined in (2). For $\alpha \in(0,1]$ derivative, let the fractional derivative of variable $\Phi(t)$ be

$$
{ }_{C} D_{t}^{\alpha} \Phi(t)=\phi(\Phi(t), t) .
$$

Utilizing the one of most common numerical methods, the L1 scheme $[22,23,24,21]$, the numerical approximation of the FOD of $\Phi(t)$ is

$$
{ }_{C} D_{t}^{\alpha} \Phi(t) \approx \frac{(d t)^{-\alpha}}{\Gamma(2-\alpha)}\left[\sum_{\rho=0}^{T-1}\left[\Phi\left(t_{\rho+1}\right)-\Phi\left(t_{\rho}\right)\right]\left[(T-\rho)^{1-\alpha}-(T-1-\rho)^{1-\alpha}\right]\right] .
$$

One of the most powerful numerical methods for discretizing the Caputo-FOD in time is L1 scheme. The purpose of implementing the L1 scheme in this research study is its memory term and convergence rate. Memory term is also explicitly present in other numerical methods, but this memory integration term is more clearly defined in the L1 scheme. Considering (10) and (11) together, the numerical solution of Eq. (10) is as follows:

$$
\Phi\left(t_{T}\right) \quad \approx \quad{ }_{C} D_{t}^{\alpha} \Gamma(2-\alpha) H(\Phi(t), t)+\Phi\left(t_{T-1}\right)-\left[\sum_{\rho=0}^{T-2}\left[\Phi\left(t_{\rho+1}\right)-\Phi\left(t_{\rho}\right)\right]\left[(T-\rho)^{1-\alpha}-(T-1-\rho)^{1-\alpha}\right]\right] .
$$

Therefore, the solution of the FOD (fractional-order derivative) can be defined as the difference between the Markov term and the memory trace. The Markov term weighted by the Gamma function is as follows:

$$
\text { Markov term }={ }_{C} D_{t}^{\alpha} \Gamma(2-\alpha) H(\Phi(t), t)+\Phi\left(t_{T-1}\right) .
$$

The memory trace ( $\Phi$-memory trace since it is related to variable $\Phi(t))$ is

$$
\text { Memory trace }=\sum_{\rho=0}^{T-2}\left[\Phi\left(t_{\rho+1}\right)-\Phi\left(t_{\rho}\right)\right]\left[(T-\rho)^{1-\alpha}-(T-1-\rho)^{1-\alpha}\right] .
$$

The memory trace is capable of integrating all past activities and takes into account the long-term history of the system. For $\alpha=1$, the memory trace is o for any time t. Memory trace dynamics is highly dependent on time. When the fractional-order $\alpha$ is decreased from the 


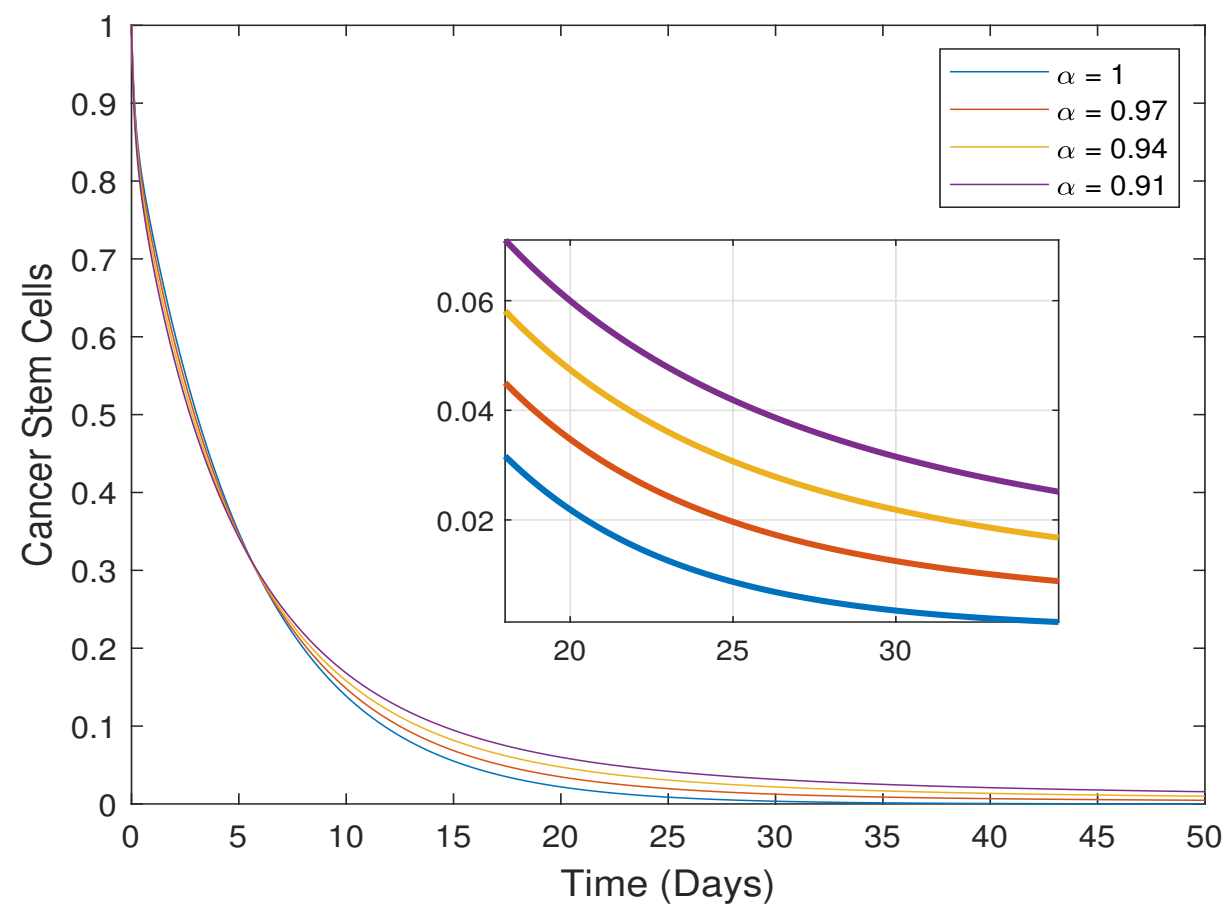

Figure 1. Change of the cancer stem cells over time for the varying fractional-order derivative

unit, the memory trace nonlinearly increases from 0 . Hence, the fractional-order system dynamics quite different from the integer order dynamics.

\section{Numerical simulations and data analysis}

In this section, for the system (2), the numerical solutions are achieved using the Adams-Bashforth-Moulton Predictor-Corrector method [34] for the parameters in Table 1. With the help of numerical simulations, we have investigated the effects of changes in parameters on the system (2) and how different values of the fractional derivative $\alpha$ affect the behavior of the system. The parameter values that have been used for numerical simulations are given in Table 1. In Fig. 1, the variation of cancer stem cells with time for different fractional derivatives have been observed. As the $\alpha$ decreases from 1 , that is, in the case of the Caputo fractional derivative, it takes a longer time for the stem cells to reach the equilibrium point. In Fig. 2, the change of tumor cells with time for different fractional derivatives have been observed. It has been seen that tumor cells disappear in a short time for the integer order case. In addition, since the fractional derivative decreases from 1 to zero (does not equal to zero), the amount of decrease in tumor cells per unit time also decreases. In Fig. 3 , it has been seen that the concentration of effector cells decreases in a short time, then their concentration suddenly increases and then reaches the equilibrium point. In addition, as $\alpha$ decreases from 1, it takes a longer time for effector cells to reach the equilibrium point. In Fig. 4, the variation of chemotherapy concentration drug with time for different fractional derivatives has been illustrated. It is understood from Fig. 4 that, the fractional order predicts more chemotherapy concentration drugs. Moreover, in Figs. 5,6,7, the changes of tumor cells and cancer stem cells with time have been investigated for different values of parameters. It is understood from Fig. 5 that as the $\gamma_{2}$ (decay rate of chemotherapy drug) increases, there is a significant increase in the number of tumor stem cells. In addition, we vary the parameter $k_{s}$ and keep other parameters fixed in order to explore the effects of this parameter in Fig. 6. From Fig. 6, it has been shown that as $k_{S}$ decreases, the number of stem cells also increases. In addition, it is clear from Fig. 7 that as $k_{T}$ decreases, the number of tumor cells also increases. We also explore the effect of the memory trace in Figs. 8,9,10,11. One can conclude that when $\alpha=1$, the memory effect in the system is zero and as the $\alpha$ increases to 1 the memory effect of the system also emerges.

\section{Results and discussion}

In this paper, we have considered the Caputo fractional order cancer-immune system model that is given as a system of fractional differential equations (2) which have Caputo fractional derivative. We explore the local asymptotic stability of the tumor-free and tumor-infection fixed points of the system and we show that the equilibrium points of the model (2) is asymptotically stable under some certain conditions. Then, we have examined the existence and uniqueness of the solution. Moreover, we have achieved the numerical simulations to verify the theoretical results. In order to explore the effects of variation of the fractional order derivative and to examine the behavior of the system, we have obtained the figures for different $\alpha$ values. It is seen that as $\alpha$ decreases from 1 , the cells reach the equilibrium points faster. In addition, we have investigated the effect of the memory trace, which is very important for biological models. When examining the effects of the memory trace, it is seen that there is no memory effect for $\alpha=1$. However, as $\alpha$ decreases from 1 , the memory effect of the system emerges. From the figures, we have concluded that the Caputo fractional derivative gives more realistic results than integer order derivatives. Although, there have been many studies that discuss the tumor-immune interaction in the literature, our model differs from them in terms of exploring the interaction between stem cells, tumor cells, effector cells and chemotherapy concentration drugs. In addition, also, it differs from other models in terms of the mathematical studies presented above. 


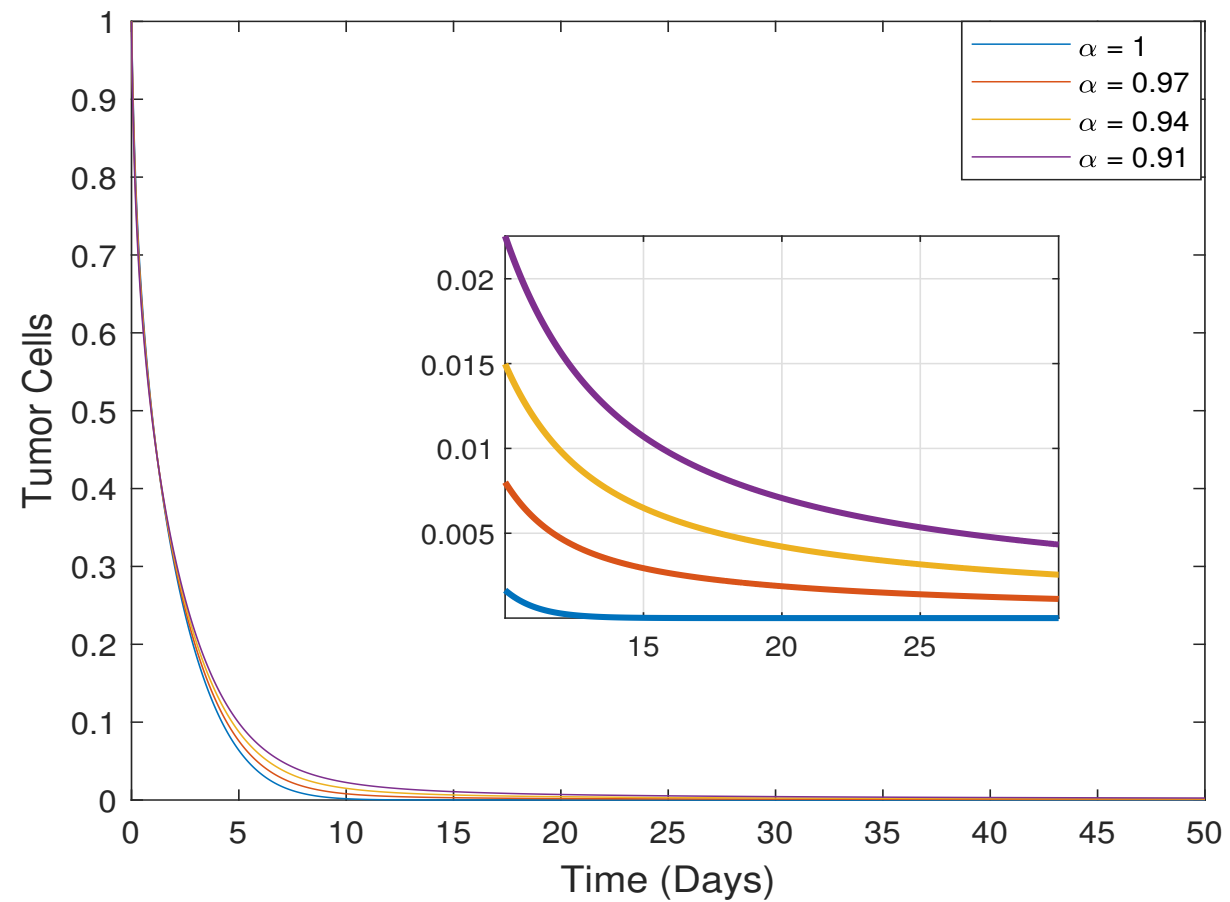

Figure 2. Change of the Tumor Cells over time for the varying fractional-order derivative

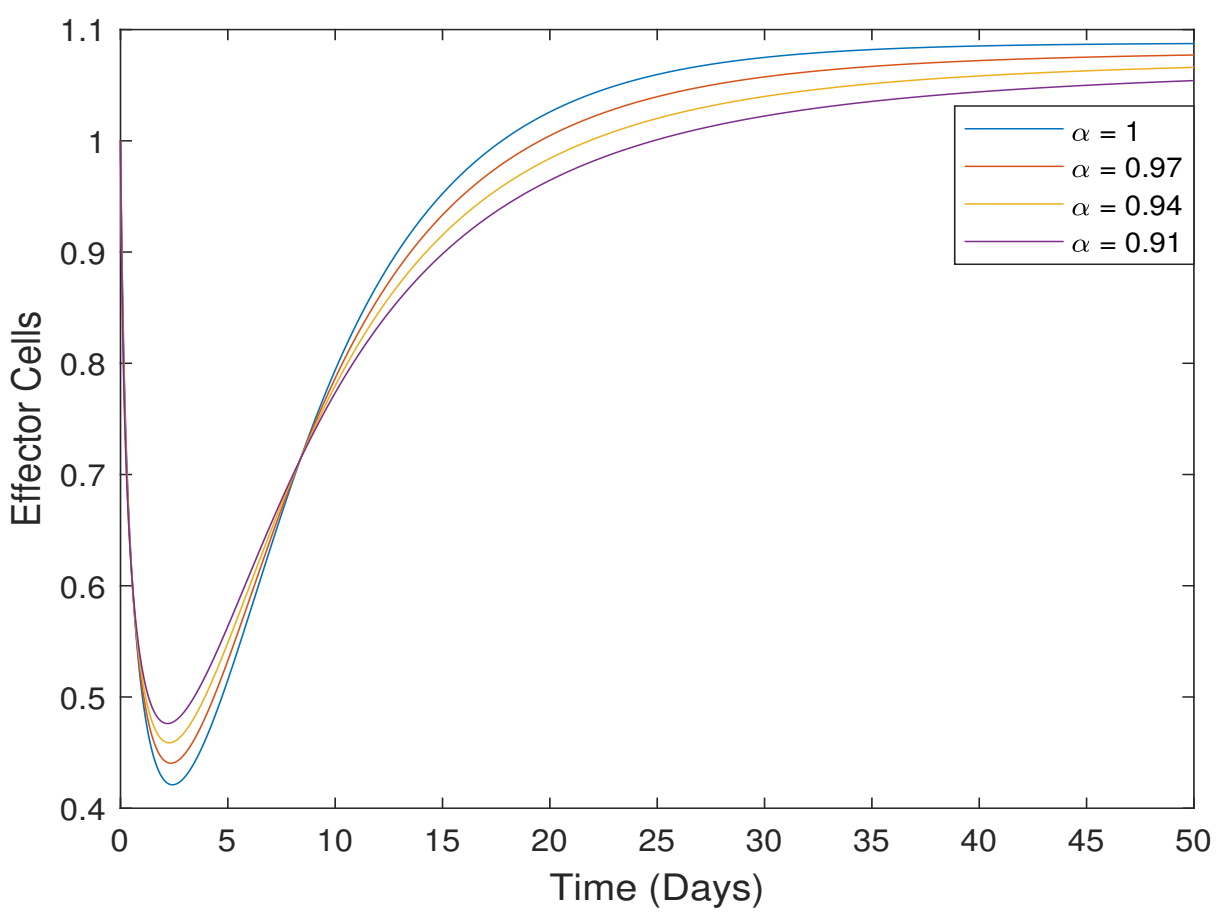

Figure 3. Change of the effector cells over time for the varying fractional-order derivative 


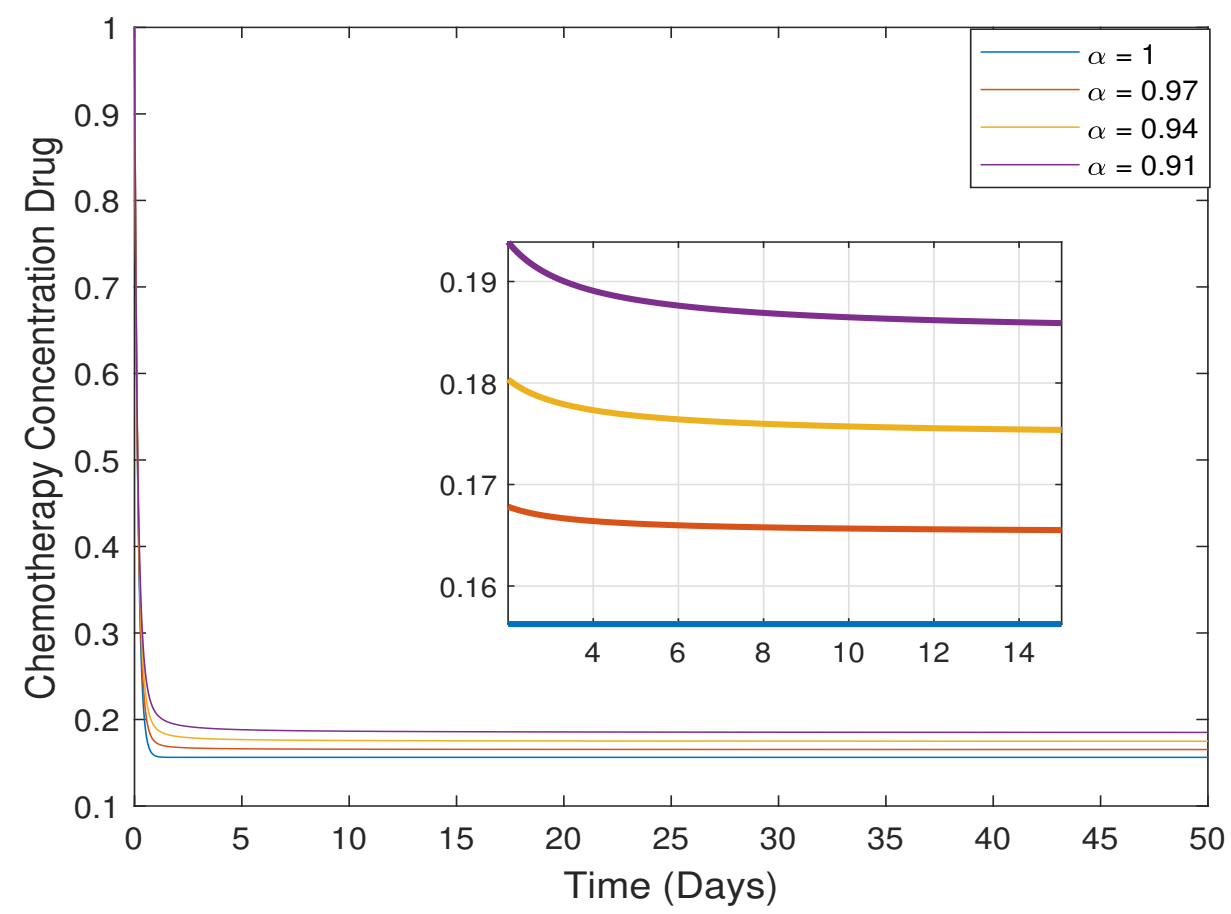

Figure 4. Change of the Chemotherapy drug concentration over time for the varying fractional-order derivative

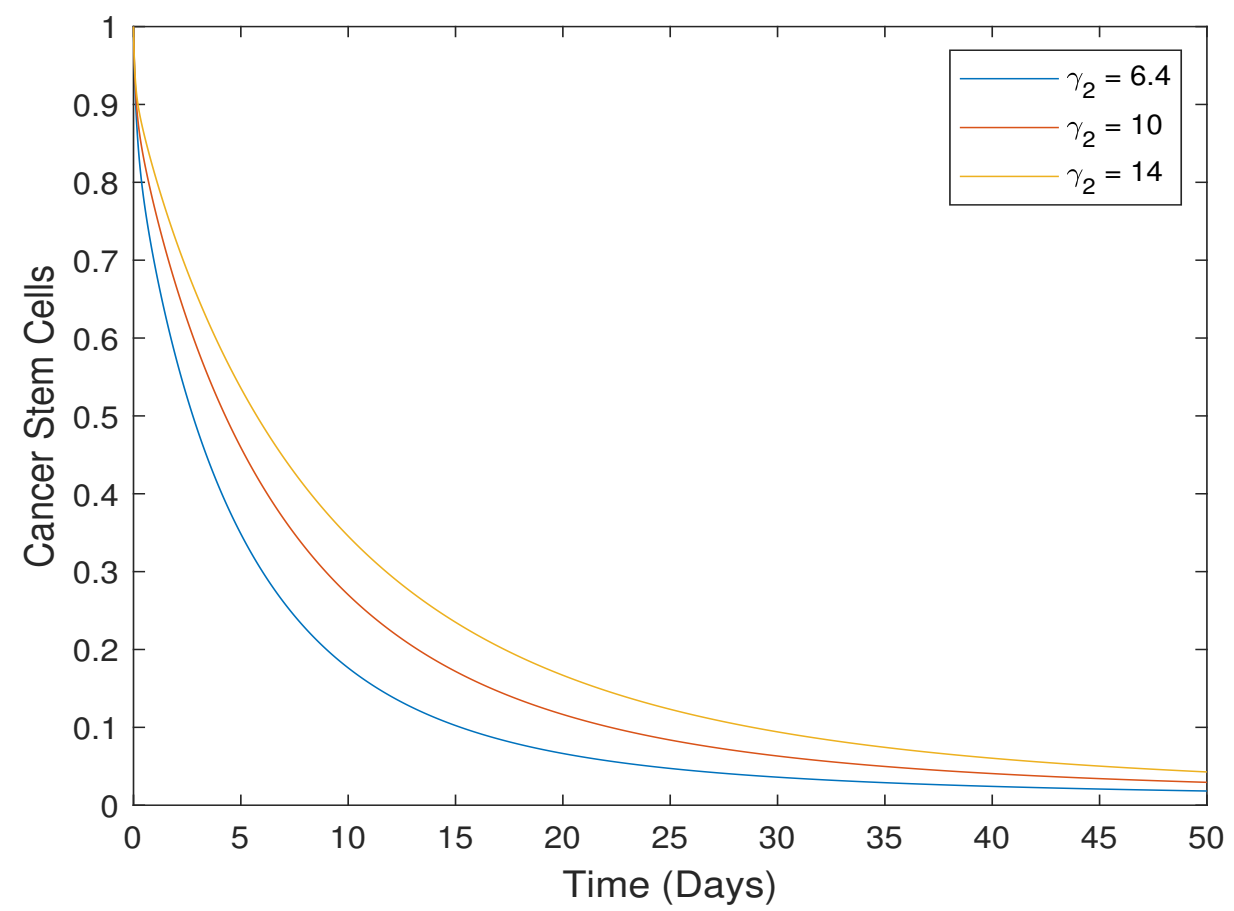

Figure 5. Change of the stem cells over time for the different $\gamma_{2}$ values, $\alpha=0.9$ 


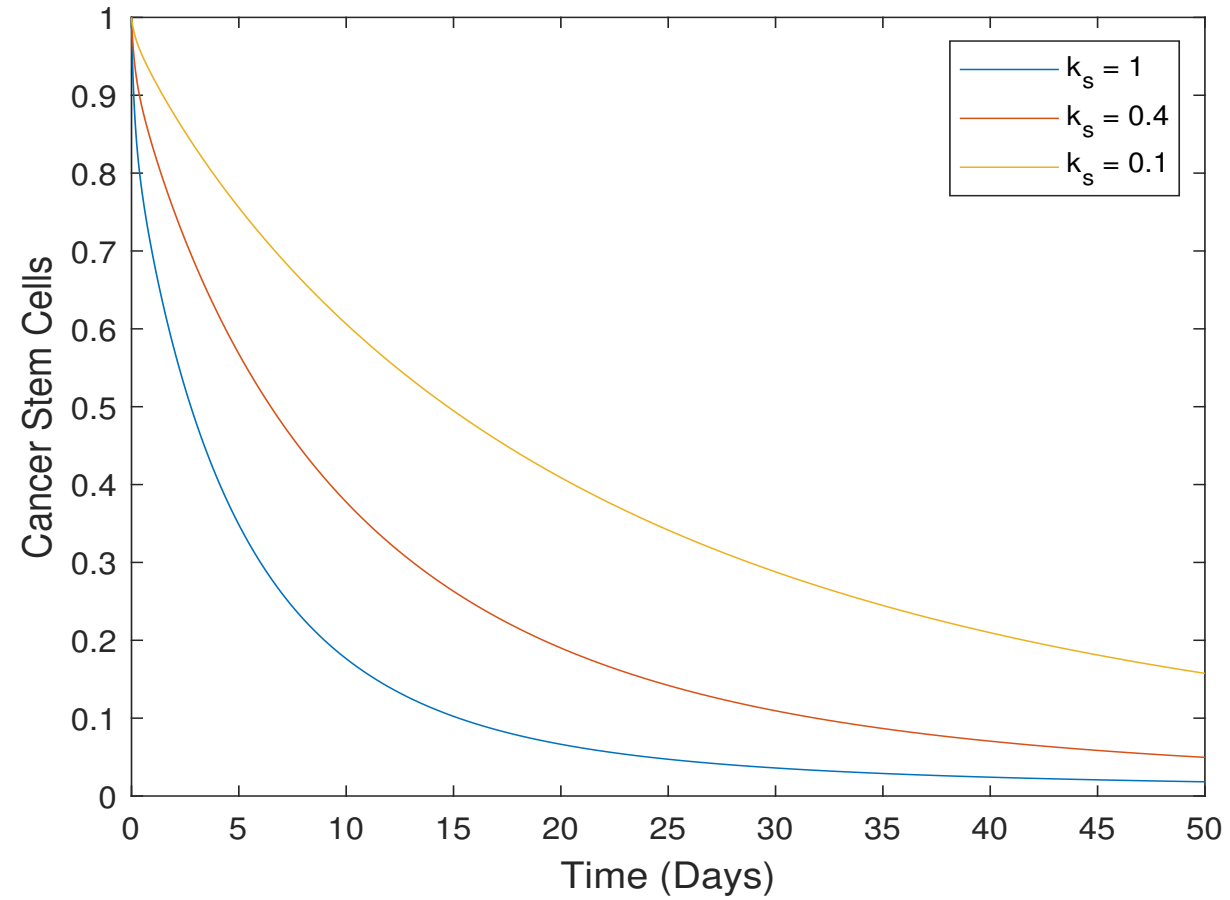

Figure 6. Change of the stem cells over time for the varying $k_{s}$ values, $\alpha=0.9$

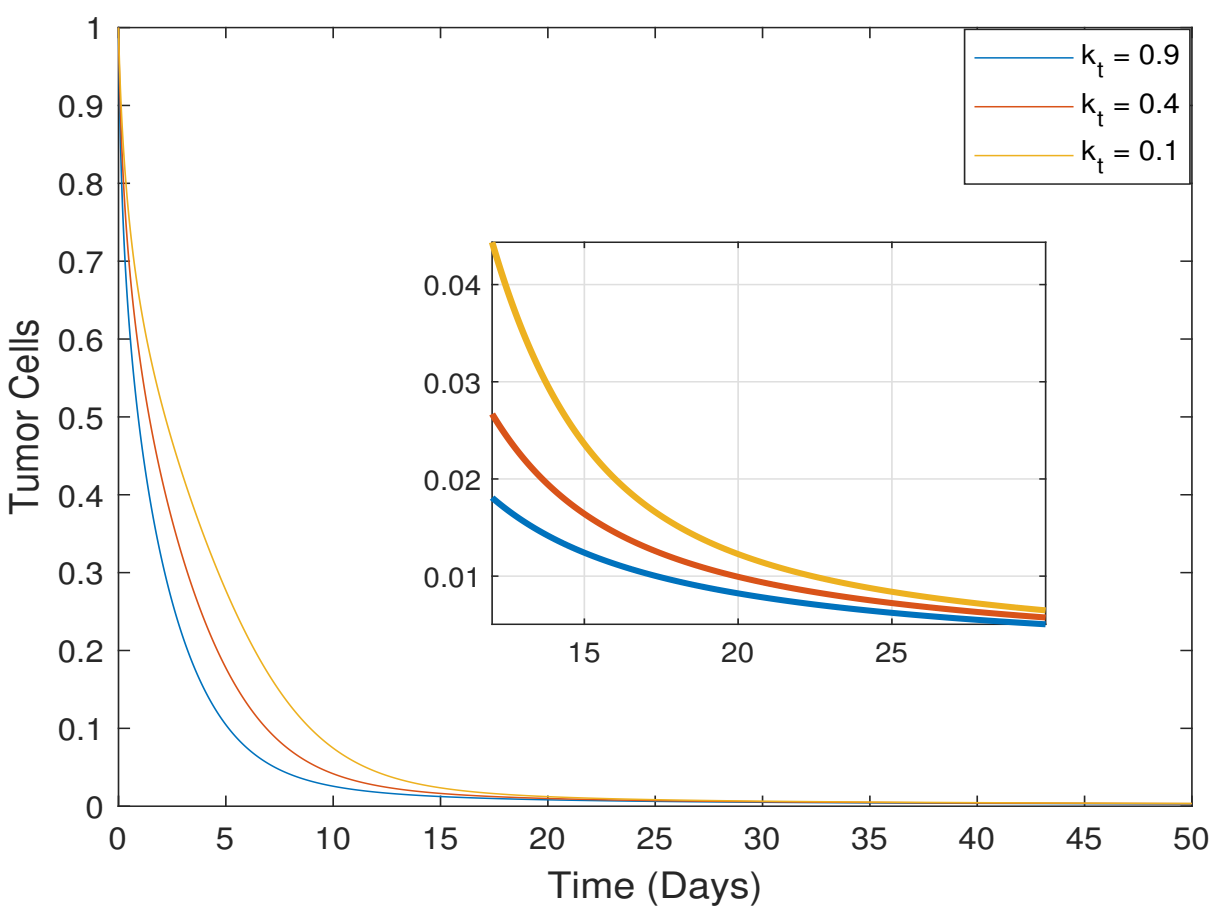

Figure 7. Change of the tumor cells over time for the varying $k_{t}$ values, $\alpha=0.9$ 


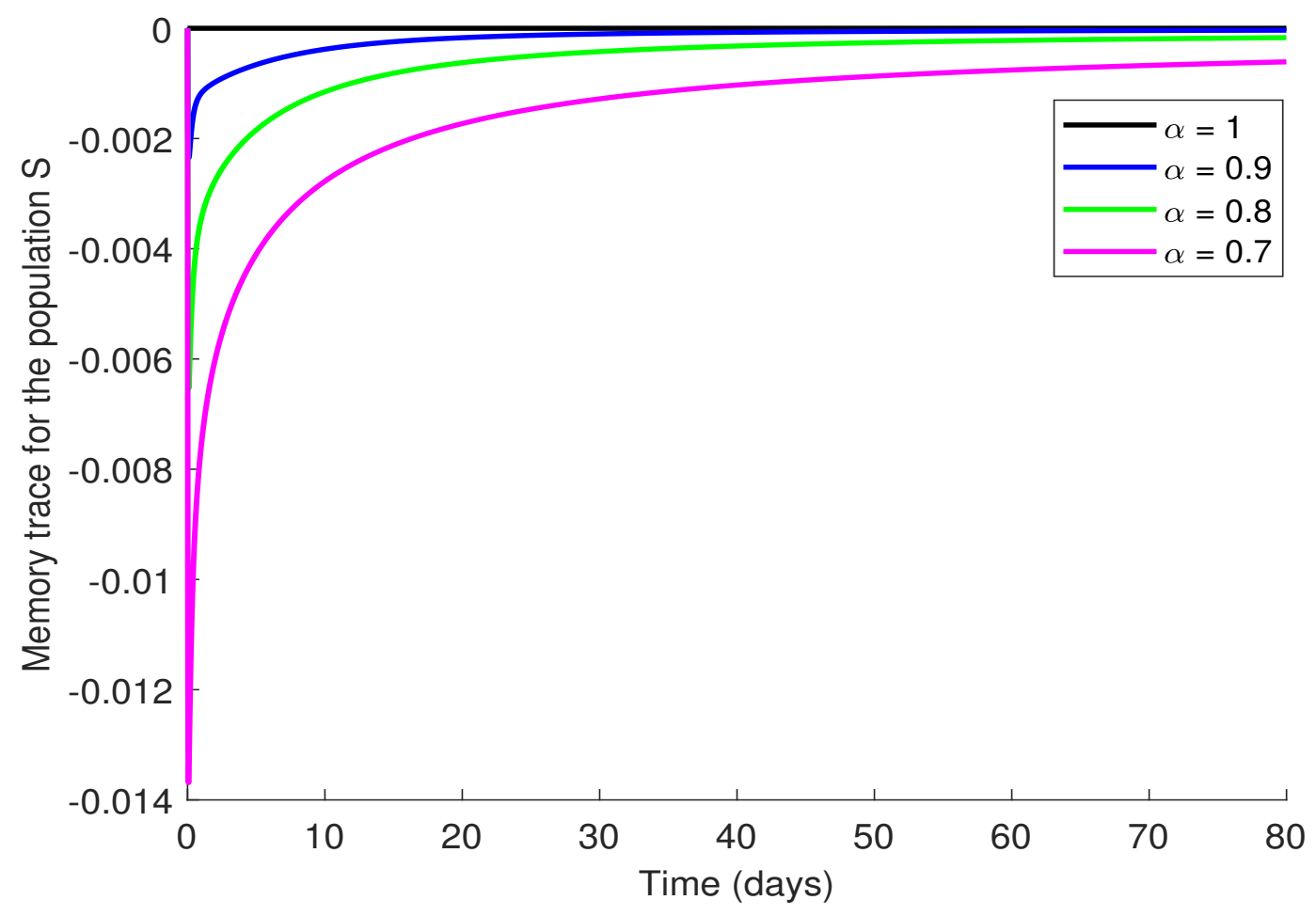

Figure 8. Change of the stem cells over time for the varying fractional-order derivative

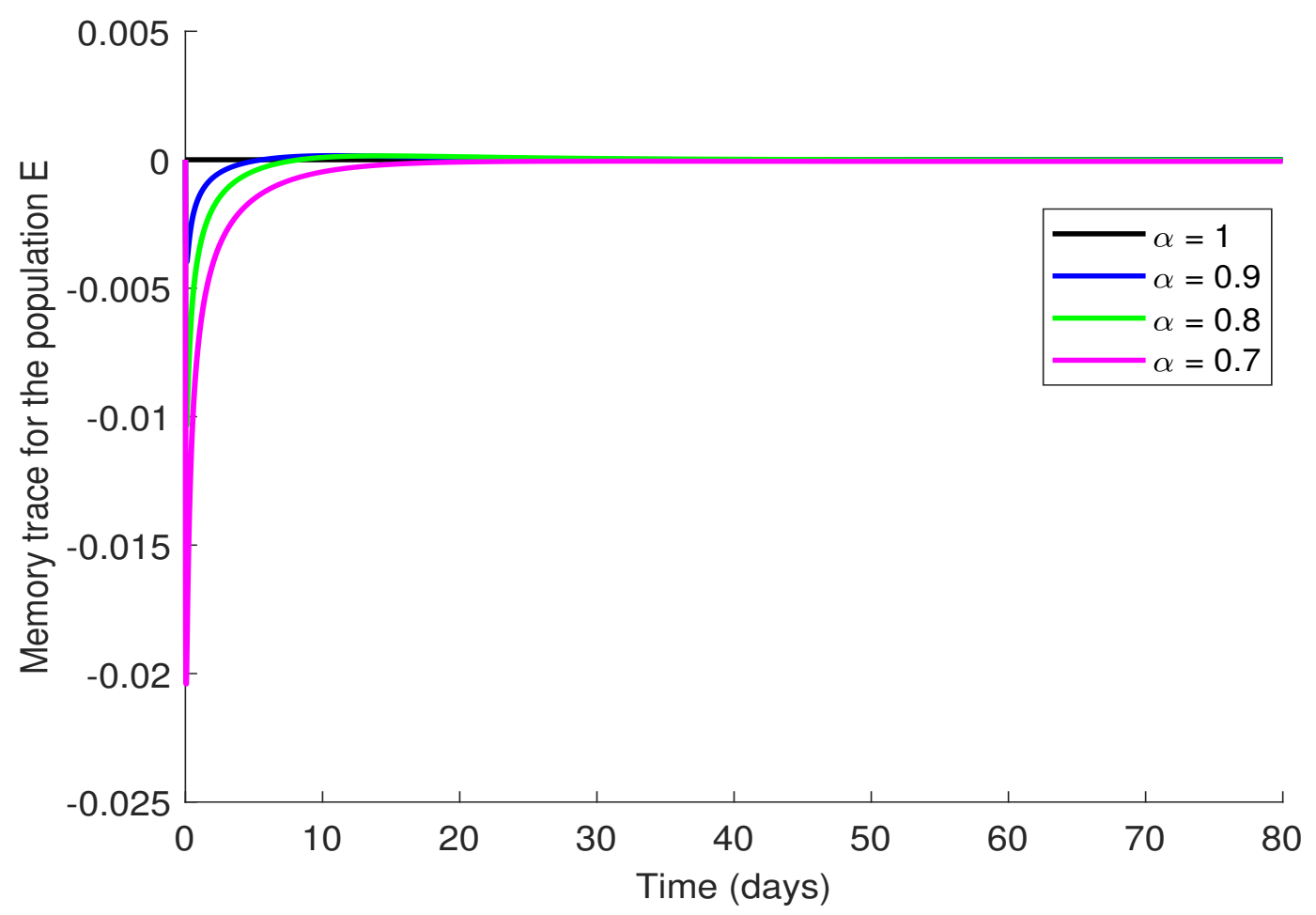

Figure 9. Change of the effector cells over time for the varying fractional-order derivative 


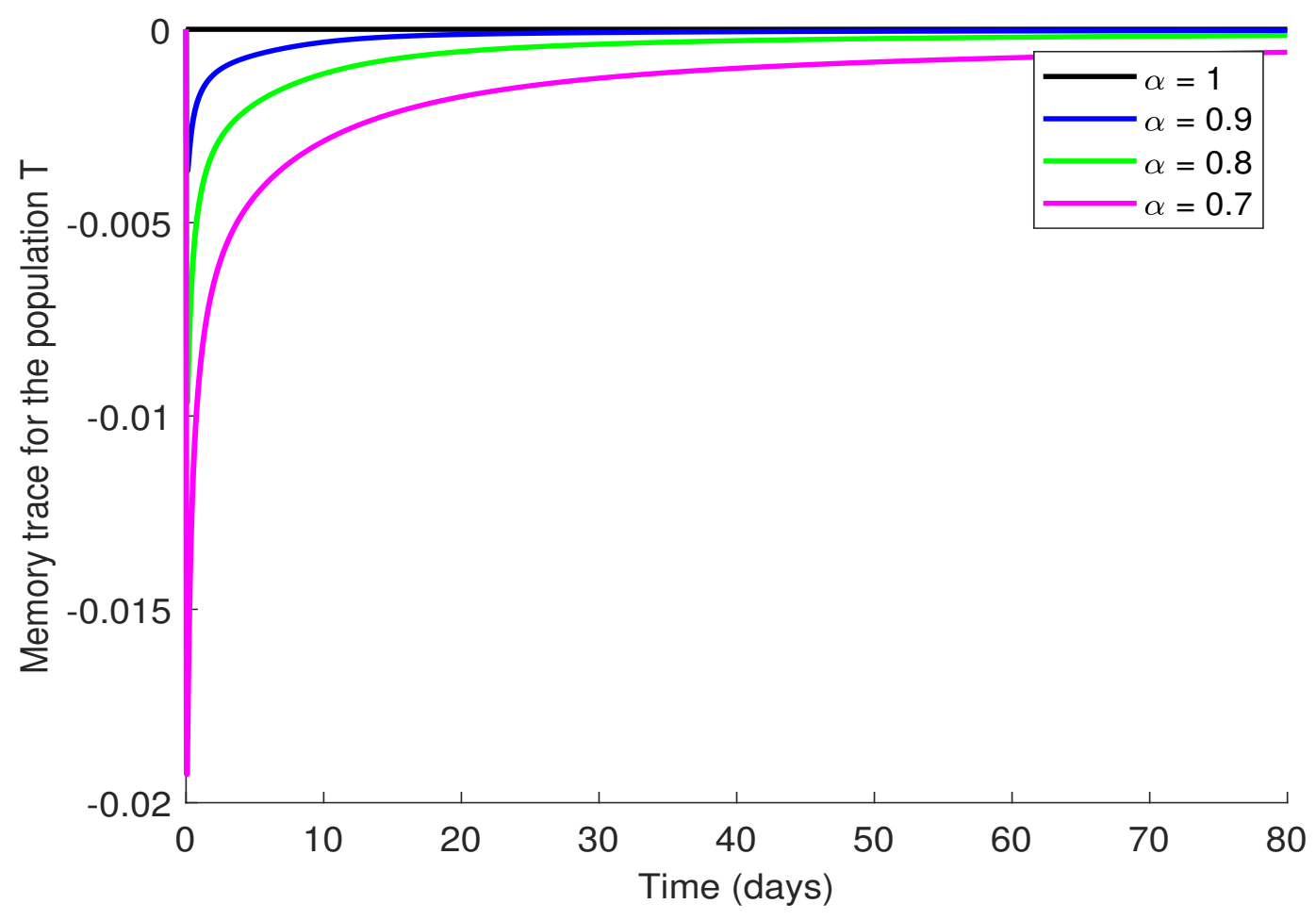

Figure 10. Change of the tumor cells over time for the varying fractional-order derivative

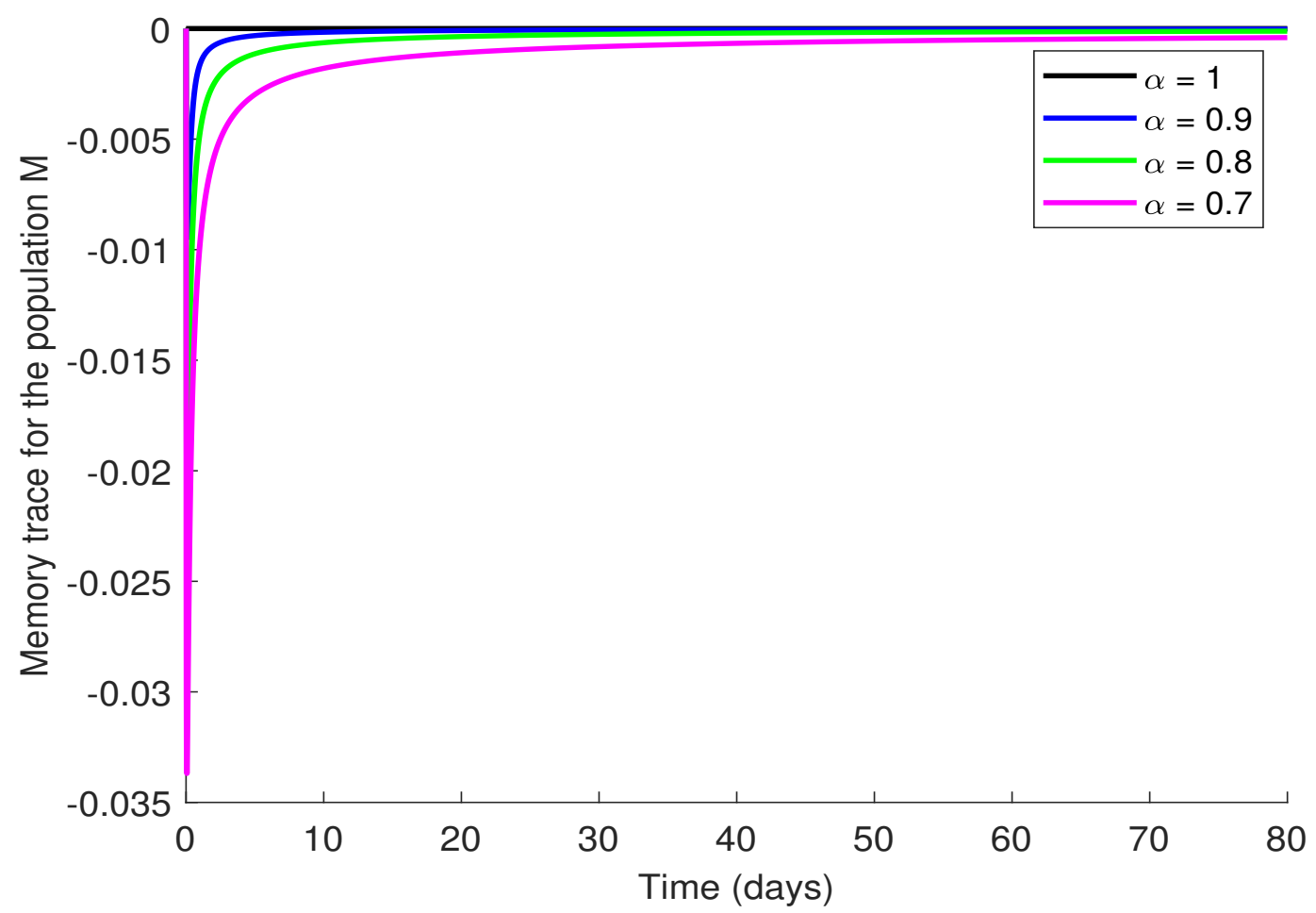

Figure 11. Change of the chemotherapy drug concentration over time for the varying fractional-order derivative 
Chemotherapy concentration drug plays an important role in the prevention of tumor growth. According to the results, if the chemotherapy concentration drugs are high, then the tumor cells undergo a considerable loss. When the simulation results have been examined, it has been observed that as $\alpha$ changes, the stem cells, the number of tumor cells, number of effector cells and chemotherapy concentration drug also change significantly. We hope that this study will make very high contributions to academics both dealing with mathematics and working in the field of medicine.

\section{Declarations}

\section{Consent for publication}

Not applicable.

\section{Conflicts of interest}

The authors declare that they have no conflict of interests.

\section{Author's contributions}

F.Ö.: Conceptualization, Methodology, Investigation, Visualization, Writing, Software, Original draft preparation, Supervision, Validation, Writing-Reviewing and Editing. M.T.Ş.:Conceptualization, Writing-Original draft preparation, Software, Supervision, Validation, WritingReviewing and Editing. R.H.:Conceptualization, Visualization, Writing, Investigation, Validation, Original draft preparation, WritingReviewing and Editing. All authors discussed the results and contributed to the final manuscript.

\section{Acknowledgements}

This study was supported by Research Fund of the Erciyes University. Project Number: FDS-2021-11059.

The authors are very much thankful to the reviewers and editor for their valuable suggestions that have improved the quality of the manuscript.

\section{References}

[1] El-Gohary, A. Chaos and optimal control of cancer self-remission and tumor system steady states. Chaos, Solutions and Fractals, 37(5), 1305-1316, (2008). [CrossRef]

[2] El-Gohary, A. The chaos and optimal control of cancer model with complete unknown parameters. Chaos, Solutions and Fractals, 42(5), 2865-2874, (2009). [CrossRef]

[3] Kirschner, D., Panetta, J.C. Modeling immunotherapy of the tumor-immune interaction. Journal of mathematical biology, 37(3), 235-252, (1998). [CrossRef]

[4] Öztürk, I. \& Özköse, F. Stability analysis of fractional order mathematical model of tumor-immune system interaction. Chaos, Solitons \& Fractals, 133, 109614, (2020). [CrossRef]

[5] Hadamard, J. Essai sur l'étude des fonctions données par leur développement de Taylor (PDF). Journal de Mathématiques Pures et Appliquées, 4(8), 101-186, (1892).

[6] Li, C. \& Tao, C. On the fractional Adams method. Computers \& Mathematics with Applications, 58(8), 1573-1588, (2009). [CrossRef]

[7] Yavuz, M. \& Sene, N. Stability analysis and numerical computation of the fractional predator-prey model with the harvesting rate. Fractal and Fractional, 4(3), 35, (2020). [CrossRef]

[8] Naik, P.A., Owolabi, K.M., Yavuz, M. \& Zu, J. Chaotic dynamics of a fractional order HIV-1 model involving AIDS-related cancer cells. Chaos, Solitons \& Fractals, 140, 110272, (2020). [CrossRef]

[9] Özköse, F. \& Yavuz, M. Investigation of interactions between COVID-19 and diabetes with hereditary traits using real data: A case study in Turkey. Computers in biology and medicine, 105044, (2021). [CrossRef]

[10] Hammouch, Z., Yavuz, M. \& Özdemir, N. Numerical solutions and synchronization of a variable-order fractional chaotic system. Mathematical Modelling and Numerical Simulation with Applications, 1(1), 11-23, (2021). [CrossRef]

[11] Naik, P.A., Yavuz, M. \& Zu, J. The role of prostitution on HIV transmission with memory: A modeling approach. Alexandria Engineering Journal, 59(4), 2513-2531, (2020). [CrossRef]

[12] Naik, P.A., Yavuz, M., Qureshi, S., Zu, J., Townley, S. Modeling and analysis of COVID-19 epidemics with treatment in fractional derivatives using real data from Pakistan. The European Physical Journal Plus, 135(10), 1-42, (2020). [CrossRef]

[13] Thomson, J.A., Itskovitz-Eldor, J., Shapiro, S.S., Waknitz, M.A., Swiergiel, J.J., Marshall, V.S., Jones, J.M. Embryonic stem cell lines derived from human blastocysts. Science, 282(5391), 1145-1147, (1998). [CrossRef]

[14] Hernigou, P., Beaujean, F. Treatment of osteonecrosis with autologous bone marrow grafting. Clinical Orthopaedics and Related Research, 405, 14-23, (2002). [CrossRef]

[15] Körbling, M., Estrov, Z. Adult stem cells for tissue repair-a new therapeutic concept?. New England Journal of Medicine, 349(6), 570-582, (2003). [CrossRef]

[16] Chakrabarty, K., Shetty, R., Ghosh, A. Corneal cell therapy: with iPSCs, it is no more a far-sight. Stem cell research \& therapy, 9(1), 1-15, (2018). [CrossRef]

[17] Alqudah, M.A. Cancer treatment by stem cells and chemotherapy as a mathematical model with numerical simulations. Alexandria Engineering Journal, 59, 1953-1957, (2020). [CrossRef]

[18] Naik, P.A., Zu, J. \& Naik, M. Stability analysis of a fractional-order cancer model with chaotic dynamics. International Journal of Biomathematics, 14(6), 2150046, (2021). [CrossRef] 
[19] Naik, P.A. Global dynamics of a fractional-order SIR epidemic model with memory. International Journal of Biomathematics, 13(8), 2050071, (2020). [CrossRef]

[20] Podlubny, I. Fractional Differential Equations. Academic Press New York, (1999).

[21] Naik, P.A., Zu, J., Owolabi, K.M. Global dynamics of a fractional order model for the transmission of HIV epidemic with optimal control. Chaos, Solitons \& Fractals, 138, 109826, (2020). [CrossRef]

[22] Jin, B., Lazarov, R. \& Zhou, Z. An analysis of the L1 scheme for the subdiffusion equation with nonsmooth data. IMA Journal of Numerical Analysis, 36(1), 197-221, (2016). [CrossRef]

[23] $\mathrm{Du}, \mathrm{M}$. \& Wang, Z. Correcting the initialization of models with fractional derivatives via history-dependent conditions. Acta Mechanica Sinica, 32(2), 320-325, (2016). [CrossRef]

[24] Magin, R.L. Fractional Calculus in Bioengineering. Redding: Begell House, (2006).

[25] Ahmed, E., El-Sayed, A.M.A., El-Saka, H.A.A. Equilibrium points, stability and numerical solutions of fractional-order predator-prey and rabies models. Journal of Matematical Analysis and Applications, 325(1), 542-553, (2007). [CrossRef]

[26] El-Sayed, A.M.A., El-Mesiry, A.E.M., El-Saka, H.A.A. On the fractional- order logistic equation. Applied Mathematics Letters, (20), 817-823, (2007). [CrossRef]

[27] Petras, I. Fractional-Order Nonlinear Systems: Modeling, Analysis and Simulation. Springer Berlin, (2011).

[28] Baisad, K., Moonchai, S. Analysis of stability and hopf bifurcation in a fractional Gauss-type predator-prey model with Allee effect and Holling type-III functional response. Advances in difference equations, 2018(1), 1-20, (2018). [CrossRef]

[29] Daşbaşı, B., Öztürk, İ. \& Özköse, F. Çoklu Antibiyotik Tedavisiyle Bakteriyel Rekabetin Matematiksel Modeli ve Kararlılık Analizi. Karaelmas Science and Engineering Journal, 6(2), 299-306, (2016). [CrossRef]

[30] Bozkurt, F. \& Özköse, F. Stability analysis of macrophage-tumor interaction with piecewise constant arguments. In AIP Conference Proceedings, (Vol. 1648, No. 1, p. 850035), AIP Publishing LLC, (2015, March). [CrossRef]

[31] Özköse, F., Yılmaz, S., Yavuz, M., Öztürk, İ., Şenel, M.T., Bağcı, B.Ş. \& Doğan, M., Önal, Ö. A fractional modelling of tumor-immune system interaction related to lung cancer with real data. The European Physical Journal Plus, (2022).

[32] Diethelm, K. An algorithm for the numerical solution of differential equations of fractional order. Electronic transactions on numerical analysis, 5(1), 1-6, (1997). [CrossRef]

[33] Diethelm, K., Ford, N.J., Freed, A.D. A predictor-corrector approch for the numerical solution of fractional differential equations. Nonlinear Dynamics, 29, 3-22, (2002). [CrossRef]

[34] Garrappa, R. On linear stability of predictor-corrector algorithms for fractional differential equations. International Journal of Computer Mathematics, 87(10), 2281-2290, (2010). [CrossRef]

Mathematical Modelling and Numerical Simulation with Applications (MMNSA) (http://www.mmnsa.org)

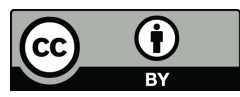

Copyright: () 2021 by the authors. This work is licensed under a Creative Commons Attribution 4.0 (CC BY) International License. The authors retain ownership of the copyright for their article, but they allow anyone to download, reuse, reprint, modify, distribute, and/or copy articles in MMNSA, so long as the original authors and source are credited. To see the complete license contents, please visit (http://creativecommons.org/licenses/by/4.0/). 